\title{
Sparse Channel Estimation for Multicarrier Underwater Acoustic Communication: From Subspace Methods to Compressed Sensing
}

\author{
Christian R. Berger, Member, IEEE, Shengli Zhou, Member, IEEE, James C. Preisig, Member, IEEE, and \\ Peter Willett, Fellow, IEEE
}

\begin{abstract}
In this paper, we investigate various channel estimators that exploit channel sparsity in the time and/or Doppler domain for a multicarrier underwater acoustic system. We use a path-based channel model, where the channel is described by a limited number of paths, each characterized by a delay, Doppler scale, and attenuation factor, and derive the exact inter-carrierinterference (ICI) pattern. For channels that have limited Doppler spread we show that subspace algorithms from the array processing literature, namely Root-MUSIC and ESPRIT, can be applied for channel estimation. For channels with Doppler spread, we adopt a compressed sensing approach, in form of Orthogonal Matching Pursuit (OMP) and Basis Pursuit (BP) algorithms, and utilize overcomplete dictionaries with an increased path delay resolution. Numerical simulation and experimental data of an OFDM block-by-block receiver are used to evaluate the proposed algorithms in comparison to the conventional least-squares (LS) channel estimator. We observe that subspace methods can tolerate small to moderate Doppler effects, and outperform the LS approach when the channel is indeed sparse. On the other hand, compressed sensing algorithms uniformly outperform the LS and subspace methods. Coupled with a channel equalizer mitigating ICI, the compressed sensing algorithms can effectively handle channels with significant Doppler spread.
\end{abstract}

Index Terms-Basis Pursuit, Doppler spread, ESPRIT, ICI, MUSIC, OFDM, Orthogonal Matching Pursuit.

\section{INTRODUCTION}

$\mathbf{U}$ NDERWATER acoustic (UWA) communication and networking has been under extensive investigation in recent years [1]-[4]. At the physical layer, UWA channels pose grand challenges for effective communications, featuring long delay spreads and significant Doppler effects due to internal waves,

Manuscript submitted May 25, 2009, revised September 13, 2009, accepted October 29, 2009. C. Berger, S. Zhou, and P. Willett are supported by ONR grants N00014-09-10613, N00014-07-1-0805, and N00014-09-1-0704. This work was presented in part at the 2009 MTS/IEEE OCEANS Conference in Bremen, Germany, and at the 2009 IEEE Intl. Workshop on Signal Proc. Adv. in Wireless Comm. in Perugia, Italy. The associate editor coordinating the review of this manuscript and approving it for publication was Prof. Roberto Lopez-Valcarce.

C. Berger was with the Department of Electrical and Computer Engineering, University of Connecticut. He is now with the Department of Electrical and Computer Engineering, Carnegie Mellon University, Pittsburgh, PA 15213 USA (e-mail: crberger@ece.cmu.edu)

S. Zhou, and P. Willett are with the Department of Electrical and Computer Engineering, University of Connecticut, 371 Fairfield Way U-2157, Storrs, CT 06269, USA (email: \{shengli, willett\}@engr.uconn.edu).

J. Preisig is with the with the Department of Applied Ocean Physics and Engineering, Woods Hole Oceanographic Institution, Woods Hole, MA 02543, USA (email: jpreisig@whoi.edu).

Digital Object Indentifier 00.0000/TSP.2009.000000 platform and sea-surface motion [5]. The long channel delay spread leads to significant inter-symbol-interference (ISI) in single-carrier transmissions [6]. The receiver complexity for channel equalization becomes a major burden when the symbol rate increases. Multicarrier approaches like orthogonal frequency division multiplexing (OFDM) can equalize the channel at low complexity, but the aforementioned Doppler effects destroy the orthogonality of the sub-carriers and lead to inter-carrier-interference (ICI).

The combination of large delay spread and significant Doppler effects qualify UWA channels as doubly (time- and frequency-) spread channels. One known approach to this class of channels is the use of a basis expansion model (BEM) to reflect the time-varying nature of the UWA channel, see e.g., [7]-[9]. Even though the time-varying nature of channels can be modeled arbitrarily well this way, it also tremendously increases demands on channel estimation, as the number of unknowns that need to be estimated increases correspondingly. The only remedy to this challenge, is to exploit the fact that UWA channels are naturally sparse, meaning that most channel energy is concentrated in a few delay and/or Doppler values [10], [11].

Sparse channel estimation for linear time-invariant (LTI) channels has been extensively studied for frequency selective radio channels based on, e.g., subspace fitting [12], model order fitting using a generalized Akaike information criterion [13], zero-tap detection [14], or Monte Carlo Markov Chain methods [15]. More recently, advances in the new field of compressive sensing [16]-[19] have led to numerous applications on sparse channel estimation, e.g., sparseness in delay only [20]-[27] and very recently for sparseness in delay and Doppler [28]-[31]. Specifically on UWA channels, the matching pursuit (MP) algorithm and its variants have been used both in [10], [32] for a single carrier system and in [33] for a multicarrier system.

We in this paper deal with sparse channel estimation for multicarrier systems. We focus on our previously used OFDM design [34]-[36] using a block-by-block receiver, where each OFDM symbol is separately, coherently demodulated based on pilot subcarriers inserted among the data subcarriers. The contributions of this paper are the following:

- We suggest the use of a path-based channel model, amenable to sparse estimation, where the UWA channel is parameterized by a number of distinct paths, each 
characterized by a triplet of delay, Doppler rate, and path attenuation. We derive the exact ICI formulation at the output of the block-by-block OFDM receiver after proper time-domain Doppler compensation.

- We link well known algorithms from the array processing literature to the sparse channel estimation problem, namely Root-MUSIC and ESPRIT [37]. These algorithms can be applied when the channel has limited Doppler spread, where the residual ICI is treated as additional noise after proper Doppler compensation.

- We use compressed sensing techniques, specifically Orthogonal Matching Pursuit (OMP) and Basis Pursuit $(\mathrm{BP})^{1}$ algorithms, to deal with channels with larger Doppler spread. Relative to existing work based on baseband channel models, we utilize dictionaries with finer delay and Doppler resolutions.

- We use extensive numerical simulation and experimental data to investigate the performance of the proposed sparse channel estimators.

The experimental data was recorded as part of the GLINT'08 experiment in the Mediterranean, south of the island Elba, Italy, in July 2008, and as part of the SPACE'08 experiment off the coast of Martha's Vineyard, MA, from Oct. 14 to Nov. 1, 2008. We have the following observations.

- Root-MUSIC and ESPRIT channel estimators outperform the conventional least-squares (LS) scheme on sparse channels, but perform worse when most energy arrives as "diffuse" multipath.

- Both OMP and BP can well handle sparse and diffuse multipath, performing uniformly the best, with BP having a slight edge over OMP.

- On channels with mild Doppler spread, receivers that operate in an ICI-ignorant manner, can achieve sufficient performance and still take advantage of the sparsity in the delay profile.

- Using compressive sensing algorithms, in particular BP, in conjunction with an ICI-aware receiver leads to drastic performance improvement in channels with severe Doppler spread.

The rest of this paper is as follows. In Section II we introduce the signal model. In Sections III and IV we present the subspace and compressed sensing algorithms, respectively. In Sections V and VI we use numerical simulation to investigate effects of time resolution and Doppler spread on channel estimation performance. Section VII contains experimental results, and we conclude in Section VIII.

Notation: We will use the following notations throughout the paper: Column vectors and matrices will be denoted by lower case, $\mathbf{x}$, and upper case, $\mathbf{A}$, bold face symbols respectively. $\mathbf{A}^{T}, \mathbf{A}^{H}$ denote the transpose and the Hermitian, the complex conjugate transpose. The Moore-Penrose pseudo inverse is denoted as $\mathbf{A}^{\dagger}$.

\footnotetext{
${ }^{1}$ When refering to $\mathrm{BP}$, we always consider the solution to the following convex optimation problem $\min _{\mathbf{x}} \frac{1}{2}\|\mathbf{A x}-\mathbf{z}\|^{2}+\tau\|\mathbf{x}\|_{1}$ that takes explicitly into account the noisy nature of the observations. This formulation is sometimes referred to as $l_{2}-l_{1}$ or Basis Pursuit Denoising (BPDN), and the solution will be equivalent to that of the Least Absolute Shrinkage and Selection Operator (LASSO) under appropriate parameterization, see e.g. [38].
}

\section{SySTEM ModeL}

We consider zero-padded (ZP) orthogonal frequency division multiplexing (OFDM) as in [34], [39]. Let $T$ denote the OFDM symbol duration and $T_{g}$ the guard interval for the ZP. The total OFDM block duration is $T^{\prime}=T+T_{g}$ and the subcarrier spacing is $1 / T$. The $k$ th subcarrier is at frequency

$$
f_{k}=f_{c}+k / T, \quad k=-K / 2, \ldots, K / 2-1,
$$

where $f_{c}$ is the carrier frequency and $K$ subcarriers are used so that the bandwidth is $B=K / T$. Let $s[k]$ denote the information symbol to be transmitted on the $k$ th subcarrier. The non-overlapping sets of data subcarriers $\mathcal{S}_{\mathrm{D}}$, pilot subcarriers $\mathcal{S}_{\mathrm{P}}$, and null subcarriers $\mathcal{S}_{\mathrm{N}}$ satisfy $\mathcal{S}_{\mathrm{D}} \cup \mathcal{S}_{\mathrm{P}} \cup$ $\mathcal{S}_{\mathrm{N}}=\{-K / 2, \ldots, K / 2-1\}$; the null subcarriers are used to facilitate Doppler compensation at the receiver (see [34]).

The transmitted signal is given by

$$
\tilde{x}(t)=\operatorname{Re}\left\{\left[\sum_{k \in \mathcal{S}_{\mathrm{D}} \cup \mathcal{S}_{\mathrm{P}}} s[k] e^{j 2 \pi \frac{k}{T} t} q(t)\right] e^{j 2 \pi f_{c} t}\right\}
$$

where $q(t)$ describes the zero-padding operation, i.e.,

$$
q(t)= \begin{cases}1 & t \in[0, T] \\ 0 & \text { otherwise }\end{cases}
$$

\section{A. Channel Model}

The underwater acoustic (UWA) time-varying channel impulse response is often defined as

$$
c(\tau, t)=\sum_{p} A_{p}(t) \delta\left(\tau-\tau_{p}(t)\right) .
$$

The continuously time varying delays are caused by motion of the transmitter/receiver as well as scattering off of the moving sea surface or refraction due to sound speed variations. The real path amplitudes change with the delays as the attenuation is related to the distance traveled as well as the physics of the scattering and propagation processes.

For the duration of an OFDM symbol, the time variation of the path delays can be reasonably approximated by a Doppler rate as,

$$
\tau_{p}(t)=\tau_{p}-a_{p} t,
$$

and the path amplitudes are assumed constant $A_{p}(t) \approx A_{p}$. Furthermore we assume that the UWA channel can be well approximated by $N_{p}$ dominant discrete paths, what we denote in the following as a "path-based" channel model. With this, the channel model can be simplified to

$$
c(\tau, t)=\sum_{p=1}^{N_{p}} A_{p} \delta\left(\tau-\left[\tau_{p}-a_{p} t\right]\right),
$$

where we specifically keep the path dependent Doppler rates $a_{p}$. The received passband signal is then

$$
\tilde{y}(t)=\sum_{p=1}^{N_{p}} A_{p} \tilde{x}\left(\left[1+a_{p}\right] t-\tau_{p}\right)+\tilde{w}(t),
$$

where $\tilde{w}(t)$ is additive noise. 


\section{B. Receiver Processing}

A two-step approach to mitigating the channel Doppler effect was proposed in [34].

1) The first step is to resample $\tilde{y}(t)$ in passband with a resampling factor $\hat{a}$ that corresponds to a rough Doppler estimate, leading to $\tilde{z}(t)$, c.f. (9).

2) The second step is to perform fine Doppler shift compensation on $z(t)$, the baseband version of $\tilde{z}(t)$, to obtain $z(t) e^{-j 2 \pi \epsilon t}$, where $\epsilon$ is the estimated residual mean Doppler shift.

The resampling can be written as the following:

$$
\begin{aligned}
\tilde{z}(t) & =\sum_{p=1}^{N_{p}} A_{p} \tilde{x}\left(\left(\frac{1+a_{p}}{1+\hat{a}}\right) t-\tau_{p}\right)+\tilde{w}(t /(1+\hat{a})), \\
& =\sum_{p=1}^{N_{p}} A_{p} \tilde{x}\left(\left(1+b_{p}\right)\left(t-\tau_{p}^{\prime}\right)\right)+\tilde{w}(t /(1+\hat{a})) .
\end{aligned}
$$

To simplify notation, we have defined the new residual Doppler rates and scaled delays

$$
\begin{aligned}
1+b_{p} & =1+\left(\frac{a_{p}-\hat{a}}{1+\hat{a}}\right)=\frac{1+a_{p}}{1+\hat{a}}, \\
\tau_{p}^{\prime} & =\frac{\tau_{p}}{1+b_{p}} .
\end{aligned}
$$

Comparing (7) with (9), we see that the received waveform after resampling is equivalent to one that passed through a channel with Doppler rates $b_{p}$. In channels with a single dominant Doppler, e.g. from platform motion, this can reduce the channel to an ICI free system. In practice this operation will let us assume that the Doppler spread is centered around zero, as a non-zero mean of the $a_{p}$ is removed by the resampling. The use of scaled delays only exchange the order of scaling and delaying in the definition of the channel impulse-response in (6).

Performing ZP-OFDM demodulation, the output $z_{m}$ on the $m$ th subchannel is

$$
z_{m}=\frac{1}{T} \int_{0}^{T+T_{g}} z(t) e^{-j 2 \pi \epsilon t} e^{-j 2 \pi \frac{m}{T} t} d t .
$$

Plugging in $z(t)$ and carrying out the integration, we simplify $z_{m}$ to

$$
z_{m}=\sum_{p=1}^{N_{p}} \frac{A_{p}}{1+b_{p}} e^{-j 2 \pi\left(f_{m}+\epsilon\right) \tau_{p}^{\prime}} \sum_{k \in \mathcal{S}_{\mathrm{D}} \cup \mathcal{S}_{\mathrm{P}}} \varrho_{m, k}^{(p)} s[k]+v_{m},
$$

where $v_{m}$ is the additive noise and

$$
\begin{aligned}
\varrho_{m, k}^{(p)} & =\frac{\sin \left(\pi \beta_{m, k}^{(p)} T\right)}{\pi \beta_{m, k}^{(p)} T} e^{j \pi \beta_{m, k}^{(p)} T}, \\
\beta_{m, k}^{(p)} & =(k-m) \frac{1}{T}+\frac{b_{p} f_{m}-\epsilon}{1+b_{p}} .
\end{aligned}
$$

Defining a stacked received vector $\mathbf{z}$, data vector $\mathbf{s}$, and noise vector $\mathbf{v}$ across all subcarriers, we can write the following input-output relationship:

$$
\mathbf{z}=\mathbf{H s}+\mathbf{v}
$$

where the channel mixing-matrix $\mathbf{H}$ has entries

$$
[\mathbf{H}]_{m, k}=\sum_{p=1}^{N_{p}} \frac{A_{p}}{1+b_{p}} e^{-j 2 \pi\left(f_{m}+\epsilon\right) \tau_{p}^{\prime}} \varrho_{m, k}^{(p)} .
$$

The channel estimation methods in this paper use a baseband formulation where each path has a complex path gain. Specifically, the mixing matrix $\mathbf{H}$ is now expressed as

$$
\mathbf{H}=\sum_{p=1}^{N_{p}} \xi_{p} \boldsymbol{\Lambda}_{p} \boldsymbol{\Gamma}_{p},
$$

where the complex path gain for the $p$ th path is

$$
\xi_{p}=\frac{A_{p}}{1+b_{p}} e^{-j 2 \pi\left(f_{c}+\epsilon\right) \tau_{p}^{\prime}},
$$

the matrix $\boldsymbol{\Gamma}_{p}$ has an $(m, k)$ th entry as $\left[\boldsymbol{\Gamma}_{p}\right]_{m, k}=\varrho_{m, k}^{(p)}$, and the matrix $\boldsymbol{\Lambda}_{p}$ is a diagonal matrix with

$$
\left[\boldsymbol{\Lambda}_{p}\right]_{m, m}=e^{-j 2 \pi \frac{m}{T} \tau_{p}^{\prime}}
$$

The formulation in (18) clearly specifies the contribution from each discrete path with delay $\tau_{p}^{\prime}$ and Doppler scale $b_{p}$ towards the channel mixing matrix that defines the ICI pattern.

\section{Subspace Methods}

When all the paths have similar Doppler scales, proper choices of $\hat{a}$ and $\epsilon$ can render $\mathbf{H}$ close to diagonal, which is the rationale for the receiver design in [34]. Specifically, the residual ICI is ignored, and $\boldsymbol{\Gamma}_{p}$ in (18) is approximated by an identity matrix.

Let us now relate this simplified setup to the direction finding problem from the array processing literature. Dividing the measurements, $z_{m}$, by the transmitted symbol on each subcarrier, $s[m]$, (in practice, only pilot subcarriers are considered, as will be clear later on), the estimated frequency responses can be collected into a vector, where we ignore the noise at this moment. Collecting the diagonal entries of $\mathbf{H}$ into a vector $\tilde{\mathbf{h}}$, we obtain

$$
\tilde{\mathbf{h}}=\sum_{p=1}^{N_{p}} \xi_{p} \mathbf{w}\left(\tau_{p}^{\prime}\right)
$$

where $\mathbf{w}\left(\tau_{p}^{\prime}\right)$ has the $m$ th entry $e^{-j 2 \pi \frac{m}{T} \tau_{p}^{\prime}}$. The formulation in (21) is thus equivalent to a direction finding problem in the array processing literature; each arrival from a certain direction has a steering vector in a similar form to $\mathbf{w}\left(\tau_{p}^{\prime}\right)$. Hence, subspace methods from array processing can be applied to identify the distinct path arrivals. Specifically, from the collected measurements, one needs to estimate the covariance matrix

$$
\mathbf{R}_{\tilde{\mathbf{h}}}=E\left[\tilde{\mathbf{h}} \tilde{\mathbf{h}}^{H}\right]=\sum_{p=1}^{N_{p}} E\left[\left|\xi_{p}\right|^{2}\right] \mathbf{w}\left(\tau_{p}^{\prime}\right) \mathbf{w}\left(\tau_{p}^{\prime}\right)^{H} .
$$

The delays $\left\{\tau_{p}^{\prime}\right\}$, in our channel estimation problem correspond to the directions of arrival in array processing, which can be identified based on eigenvalue-decomposition of the covariance matrix $\mathbf{R}_{\tilde{\mathbf{h}}}$. 
Usually, a number of OFDM symbols (let us say $I$ ) need to be observed to approximate the covariance matrix,

$$
\mathbf{R}_{\tilde{\mathbf{h}}} \approx \frac{1}{I} \sum_{i=1}^{I} \tilde{\mathbf{h}}_{i} \tilde{\mathbf{h}}_{i}^{H} .
$$

In our work, we assume a block-by-block receiver as in [34]. Hence, we need to estimate the covariance matrix based on one OFDM symbol only. This is possible via spatial smoothing (see, e.g., [40] or [37]). In a nutshell, as long as the elements of the steering vectors $\mathbf{w}\left(\tau_{p}^{\prime}\right)$ exhibit a shift invariance property, we can exchange the observation of a large array for multiple "independent" observations of a smaller array, but generated by the same $\tau_{p}^{\prime}$.

Specifically, let us assume that the pilots are spaced uniformly within each OFDM symbol, i.e., $m=\Delta, 2 \Delta, \ldots$ and introduce partial vectors $\tilde{\mathbf{h}}_{a}^{b}, \mathbf{w}_{a}^{b}$, which includes pilots $a$ through $b$ of the original vector:

$$
\mathbf{w}_{a}^{b}\left(\tau_{p}^{\prime}\right)=\left[\begin{array}{llll}
e^{-j 2 \pi \frac{a \Delta}{T} \tau_{p}^{\prime}} & e^{-j 2 \pi \frac{(a+1) \Delta}{T} \tau_{p}^{\prime}} & \cdots & e^{-j 2 \pi \frac{b \Delta}{T} \tau_{p}^{\prime}}
\end{array}\right]^{T}
$$

Therefore, we have

$$
\begin{aligned}
\tilde{\mathbf{h}}_{a+\delta}^{b+\delta} & =\sum_{p=1}^{N_{p}} \xi_{p} \mathbf{w}_{a+\delta}^{b+\delta}\left(\tau_{p}^{\prime}\right) \\
& =\sum_{p=1}^{N_{p}}\left(\xi_{p} e^{-j 2 \pi \delta \frac{\Delta}{T} \tau_{p}^{\prime}}\right) \mathbf{w}_{a}^{b}\left(\tau_{p}^{\prime}\right)
\end{aligned}
$$

which can be interpreted as a second observation of $\tilde{\mathbf{h}}_{a}^{b}$ with new amplitudes $\xi_{p} e^{-j 2 \pi \delta \frac{\Delta}{T} \tau_{p}^{\prime}}$. We can approximate the covariance matrix of size $N_{C}=b-a$ as,

$$
\mathbf{R}_{\tilde{\mathbf{h}}}^{N_{C}} \approx \frac{1}{I} \sum_{i=1}^{I} \tilde{\mathbf{h}}_{i}^{i+N_{C}}\left(\tilde{\mathbf{h}}_{i}^{i+N_{C}}\right)^{H}
$$

where $I=K / \Delta-N_{C}+1$ depends on the number of available observations (pilots). Clearly there is a trade off: a larger $N_{C}$ leads to better resolution of the $\tau_{p}^{\prime}$, while a larger $I$ approximates the covariance matrix better. In any case both dimensions have to be larger than the assumed maximum number of paths, as the rank of the covariance matrix limits the maximum number of identifiable components.

\section{A. Root-MUSIC}

We choose the unitary implementation of Root-MUSIC, to reduce computational complexity (for details see [37]). The order selection problem is solved in the following way: after matrix decomposition of the covariance matrix, we choose all eigenvectors corresponding to eigenvalues less than twice the noise variance to compose the noise space.

Once the model order $N_{p}$ and the delays $\left\{\tau_{p}^{\prime}\right\}$ are estimated, the complex path gains $\xi_{p}$ are estimated as based on the pilot subcarriers,

$$
\left\{\xi_{p}\right\}_{\mathrm{LS}}=\arg \min _{\left\{\xi_{p}\right\}} \sum_{m \in \mathcal{S}_{P}}\left|H_{m}-\sum_{p=1}^{N_{p}} \xi_{p} e^{-j 2 \pi \frac{m}{T} \tau_{p}}\right|^{2},
$$

where the $H_{m}$ are the entries of the vector $\tilde{\mathbf{h}}$. Then the channel response on the data subcarriers is reconstructed in a similar way as

$$
H_{m}=\sum_{p=1}^{N_{p}} \xi_{p} e^{-j 2 \pi \frac{m}{T} \tau_{p}} \quad m \in \mathcal{S}_{D}
$$

\section{B. ESPRIT}

As with Root-MUSIC, we choose the unitary implementation for ESPRIT, following the details in [41] or [37]. The signal space is determined complementary to the noise subspace in MUSIC; we choose all eigenvectors corresponding to eigenvalues larger or equal to twice the noise variance. To improve robustness against model mismatch (especially caused by Doppler), we solve for the unknown delay parameters $\tau_{p}^{\prime}$ using a total-least-squares (TLS) formulation. Then the channel response on the data subcarriers is determined as in Sec. III-A.

\section{Compressed Sensing}

Although $\mathbf{H}$ in (18) has $K^{2}$ entries, it is defined by $N_{p}$ triplets of $\left(\xi_{p}, b_{p}, \tau_{p}^{\prime}\right)$. Since UWA channels are sparse, the value of $N_{p}$ is small, hence, it is possible that those $N_{p}$ paths can be identified by compressed sensing methods based on only a limited number of measurements.

To facilitate implementation, we rewrite $\mathbf{z}$ as

$$
\mathbf{z}=\left[\begin{array}{lll}
\boldsymbol{\Lambda}_{1} \boldsymbol{\Gamma}_{1} \mathbf{s} & \cdots & \boldsymbol{\Lambda}_{N_{p}} \boldsymbol{\Gamma}_{N_{p}} \mathbf{s}
\end{array}\right]\left[\begin{array}{c}
\xi_{1} \\
\vdots \\
\xi_{N_{p}}
\end{array}\right]+\mathbf{v} .
$$

If the parameters $\left(b_{p}, \tau_{p}^{\prime}\right)$ were available, we could construct the $\left(K \times N_{p}\right)$-matrix in (30) and solve for the $\xi_{p}$ using least squares.

\section{A. Non-Linear Estimation via Compressed Sensing}

A brute force approach to solve (30) would be to try all possible combinations of $\left\{\left(b_{p}, \tau_{p}^{\prime}\right)\right\}_{p=1}^{N_{p}}$ and choose the solution with the best fit. Of course the fit always improves as a function of $N_{p}$, which is also unknown. Similar estimation problems have been solved using compressed sensing (see [16]-[19]). An observed signal is defined as a linear combination of an unknown number of structured signals, each defined by an unknown parameter(s). This problem is solved by constructing a so-called dictionary, made of the signals parameterized by a representative selection of possible parameters (or parameter sets). In this model, parameter sets not part of the solution will be assigned a zero weight coefficient. Since a large number of such sets is necessary to construct an accurate dictionary, most weights will be zero and the problem is sparse.

We follow this approach and choose representative sets of $\left(b, \tau^{\prime}\right)$ as,

$$
\begin{aligned}
\tau^{\prime} & \in\left\{\frac{T}{\lambda K}, \frac{2 T}{\lambda K}, \cdots, T_{g}\right\}, \\
b & \in\left\{-b_{\max },-b_{\max }+\Delta b, \cdots, b_{\max }\right\} .
\end{aligned}
$$


The discretization in $\tau^{\prime}$ is based on the assumption that after synchronization all arriving paths fall into the guard interval, where we choose the time resolution as a multiple, $\lambda$, of the baseband sampling time $T / K$, leading to $N_{\tau}=\lambda K T_{g} / T$ tentative delays. For the residual Doppler rates, we assume that they are spread around zero after compensation by $\hat{a}$, and $b_{\text {max }}$ can be chosen based on the assumed Doppler spread, with resolution $2 b_{\max } /(\Delta b)+1=N_{b}$. Hence, a total of $N_{\tau} N_{b}$ candidate paths will be searched, and we expect $N_{p} \ll N_{\tau} N_{b}$ significant paths due to the channel sparsity.

With this, we form vectors

$$
\mathbf{x}_{A}^{(i)}=\left[\xi_{1}^{(i)}, \ldots, \xi_{N_{\tau}}^{(i)}\right]^{T}
$$

corresponding to all delays associated with Doppler scale $b_{i}$, and form a stacked vector $\mathbf{x}$. The linear formulation of the problem is

$$
\begin{aligned}
\mathbf{z} & =\left[\begin{array}{lll}
\boldsymbol{\Lambda}_{1} \boldsymbol{\Gamma}_{1} \mathbf{s} & \cdots & \boldsymbol{\Lambda}_{N_{\tau} N_{b}} \boldsymbol{\Gamma}_{N_{\tau} N_{b}} \mathbf{s}
\end{array}\right]\left[\begin{array}{c}
\mathbf{x}_{A}^{(1)} \\
\vdots \\
\mathbf{x}_{A}^{\left(N_{b}\right)}
\end{array}\right]+\mathbf{v} \\
& :=\mathbf{A} \mathbf{x}+\mathbf{v}
\end{aligned}
$$

where $\mathbf{A}$ is a fat matrix with $N_{\tau} N_{b}$ columns, and most of entries of $\mathbf{x}$ are assumed to be zeros since the channel is sparse. Without the assumption that most entries are zero, the problem would be ill defined, i.e., estimation of the parameters would be impossible.

\section{B. BP and OMP Algorithms}

To solve the sparse estimation problem with the measurement model in (33), we focus on two popular algorithms:

1) Basis Pursuit: As BP we denote the solution to the following convex optimization problem,

$$
\min _{\mathbf{x}} \frac{1}{2}\|\mathbf{A} \mathbf{x}-\mathbf{z}\|^{2}+\tau\|\mathbf{x}\|_{1} .
$$

This formulation explicitly takes into account the noisy nature of the observations. For an efficient solver for the complexvalued version of this formulation see, e.g., [38]. The parameter $\tau$ has to be tuned as no analytical parameterization is available. In our experience this parameter seems quite robust against the choice of values.

2) Orthogonal Matching Pursuit: This greedy algorithm iteratively identifies one $\tau_{p}$ at a time and solves a constrained LS problem at each iteration to measure the fitting error. Details for this algorithm can be found in, e.g., [10], [42]. Since the number of paths $N_{p}$ is unknown, a stopping criterion needs to be defined carefully. We compare the residual fitting error and decrease of fitting error at each iteration to thresholds based on the noise power to determine the termination of the algorithm.

For implementation of both algorithms, it is important to consider that multiplying by the matrix $\mathbf{A}$ can be done efficiently using FFTs.

To reduce the complexity of computing the dictionary set with a large size, we choose to retain only $D$ off-diagonals on the templates $\boldsymbol{\Gamma}_{p}$, (therefore also on $\mathbf{H}$ ). This means that only ICI from $D$ directly neighboring subcarriers on each
TABLE I

PARAMETERS OF ZP-OFDM IN NUMERICAL SIMULATION AND SPACE'08 EXPERIMENT.

\begin{tabular}{|l|c|c|}
\hline carrier frequency & $f_{c}$ & $13 \mathrm{kHz}$ \\
bandwidth & $B$ & $9.77 \mathrm{kHz}$ \\
no. subcarriers & $K$ & 1024 \\
symbol duration & $T$ & $104.86 \mathrm{~ms}$ \\
subcarrier spacing & $\Delta f:=1 / T$ & $9.54 \mathrm{~Hz}$ \\
guard interval & $T_{g}$ & $24.6 \mathrm{~ms}$ \\
\hline
\end{tabular}

side are considered. The symbol vector $\mathbf{s}$ contains known pilot symbols, and zeros, but also unknown data symbols. The unknown data symbols are set to zero to compute the matrix A.

Once the channel mixing matrix is constructed, a minimum mean square error (MMSE) receiver (see e.g. [43]) is applied for data demodulation

$$
\hat{\mathbf{s}}=\left(\mathbf{H}^{H} \mathbf{H}+N_{0} \mathbf{I}\right)^{-1} \mathbf{H}^{H} \mathbf{z}
$$

( $N_{0}$ is the noise power and the symbols $s[k]$ are assumed of unit average power) followed by channel decoding for data recovery. Again, the banded matrix structure of $\mathbf{H}$ leads to reduced complexity by allowing efficient matrix inversion. The special case of $D=0$ corresponds to an ICI-ignorant receiver, where $b_{\max }$ in (32) will be set to zero correspondingly.

\section{EfFect of Time Resolution on Sparse Channel ESTIMATION}

To investigate channels that are sparse in the time domain, we will first focus on linear time invariant channels, and will consider channels with Doppler spread in Section VI. This is motivated by the fact that previous work on sparse channel estimation has focused only on channels that are sparse in the equivalent discrete baseband representation. Although this representation can capture the full channel effect, corresponding to a complete basis, considering an increased time resolution will render a more sparse channel representation, which in turn improves channel estimation accuracy.

\section{A. Simulation Setup}

For purpose of numerical simulation, we approximate the continuous time operations in (12) with a sampling rate being twice the bandwidth. We start with a sparse channel with $N_{p}=$ 15 discrete paths, where the inter-arrival times are distributed exponentially with mean $E\left[\tau_{p+1}-\tau_{p}\right]=1 \mathrm{~ms}$. Hence, the average channel delay spread is about $15 \mathrm{~ms}$. The amplitudes are Rayleigh distributed with the average power decreasing exponentially with delay, where the difference between the beginning and the end of the guard time of $24.6 \mathrm{~ms}$ is $20 \mathrm{~dB}$.

The ZP-OFDM specifications in the numerical simulation are deliberately chosen to match the settings used in the SPACE'08 experiment. The carrier frequency, bandwidth, number of subcarriers, inter carrier spacing, and symbol interval are summarized in Table I.

The data rate, $R$, depends also on the modulation scheme and the number of subcarriers used for channel estimation. We adopt the subcarrier allocation from [36]. Out of the $K=1024$ subcarriers, there are $\left|\mathcal{S}_{\mathrm{P}}\right|=256$ subcarriers carrying pilot 


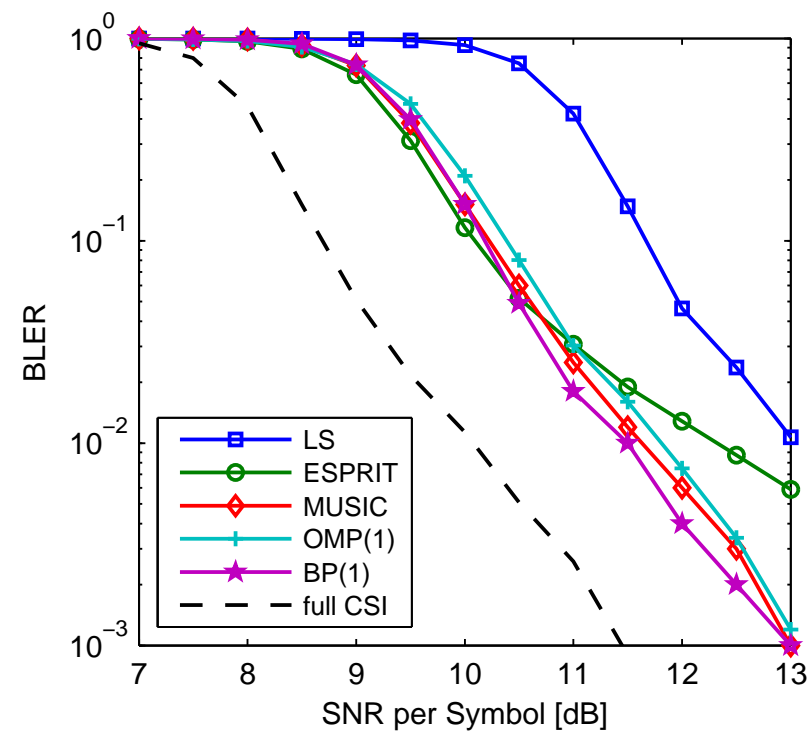

Fig. 1. All sparse channel estimation schemes significantly outperform the conventional LS estimator.

symbols, distributed on every fourth subcarrier, and $\left|\mathcal{S}_{\mathrm{N}}\right|=96$ zeros, half at the band edges and half inserted randomly between the data. The remaining 672 data subcarriers are encoded using a rate $1 / 2$ nonbinary LDPC code (see [36] for details). With a 16-QAM constellation, the spectral efficiency $\alpha$ and the data rate $R$ are

$$
\begin{aligned}
\alpha & =\frac{T}{T+T_{g}} \cdot \frac{672}{1024} \cdot \frac{1}{2} \cdot \log _{2} 16=1.1 \mathrm{bits} / \mathrm{s} / \mathrm{Hz}, \\
R & =\alpha B=10.4 \mathrm{~kb} / \mathrm{s} .
\end{aligned}
$$

We use block-error-rate (BLER) as our performance measure, which is the average number of error-free OFDM blocks after LDPC decoding. We see this as a reasonable performance criterion, since on unreliable channels such as UWA, it can be expected that there is a mechanism in place to recover lost blocks, e.g., automatic repeat-request (ARQ) or a higher layer block erasure code. In this context it has been recently shown that BLER's around $10^{-1}$ to $10^{-2}$ achieve optimal overall spectral efficiency [44], when combined with a higher layer erasure code.

\section{B. Baseband Sampling}

The compressed sensing algorithms use a dictionary only in the delay dimension (i.e., $b_{\max }=0$ ); furthermore the delay grid is at first spaced at baseband sampling rate:

$$
\tau^{\prime} \in\left\{\frac{T}{K}, \frac{2 T}{K}, \cdots, T_{g}\right\},
$$

which corresponds to $\lambda=1$. These are typical assumptions that have been made in previous work on sparse channel estimation, see [10], [20]-[27], [33]; where a) Doppler spread is ignored, and b) the channel is assumed sparse in the equivalent discrete baseband representation. We designate this implementation as $\mathrm{OMP}(1)$ and $\mathrm{BP}(1)$ to reflect the value of $\lambda$.

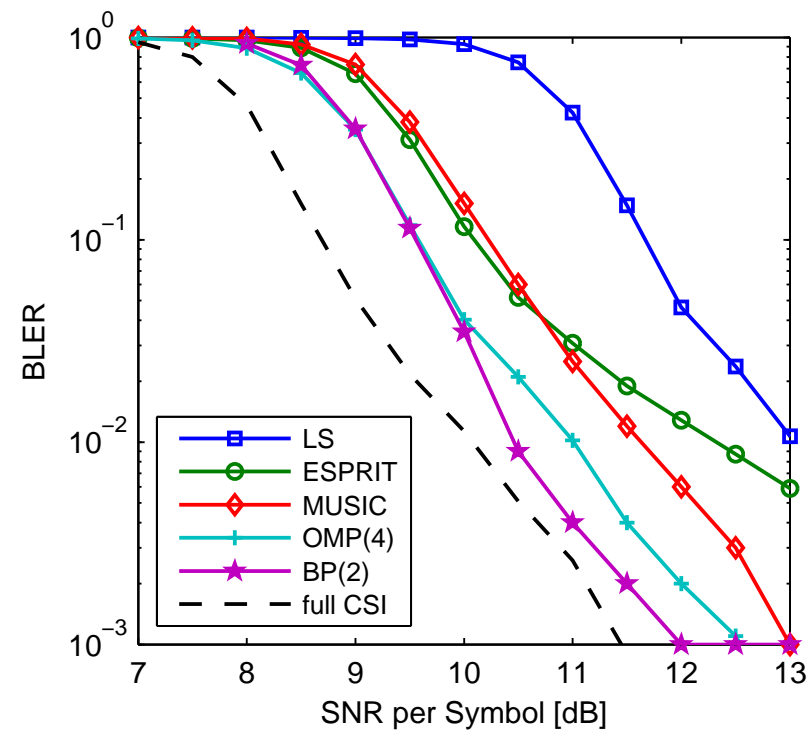

Fig. 2. The compressed sensing methods can increase their performance significantly by using dictionaries with finer delay resolution (for OMP $\lambda=4$, for $\operatorname{BP} \lambda=2$ ).

Simulation results are plotted in Fig. 1. Clearly all sparse channel estimation schemes outperform the simple leastsquares (LS) channel estimator (see [34] for details), gaining about $1.5 \mathrm{~dB}$. We also include a plot based on full channel state information (CSI) as a lower bound. All sparse channel estimation methods perform similarly well, where ESPRIT is slightly preferable for low SNR, but lags behind as SNR increases.

\section{High Time Resolution Dictionaries $\lambda>1$}

We next increase the dictionary size of the compressed sensing methods, to reflect the discrete nature of the channel in continuous time, corresponding to our path-based channel model. We find that a $\lambda>1$ increases performance significantly, but the improvement saturates quickly. We plot the same simulation with $\lambda=4$ for OMP and $\lambda=2$ for BP (see Fig. 2). Although the delays at baseband sampling $(\lambda=1)$ form a complete basis to explain the channel effect, the use of over-complete dictionaries improves performance by almost $1 \mathrm{~dB}$. As the path delays are generated from a continuous time distribution, the dictionaries with higher time resolution can explain the observations with fewer non-zero elements.

\section{Time Resolution vs. Composite Effect}

Based on our reasoning on time resolution, the subspace methods Root-MUSIC and ESPRIT should outperform the compressed sensing methods, as they inherently operate on a continuous estimation space, while the compressed sensing methods can only approximate the continuous time operation. We speculate that the super-resolution properties of subspace methods do not work well when several paths fall too close to be resolved, leading to a known bias in subspace estimators [37]. In these cases the compressed sensing methods model the composite effect, which is ultimately the rationale behind 


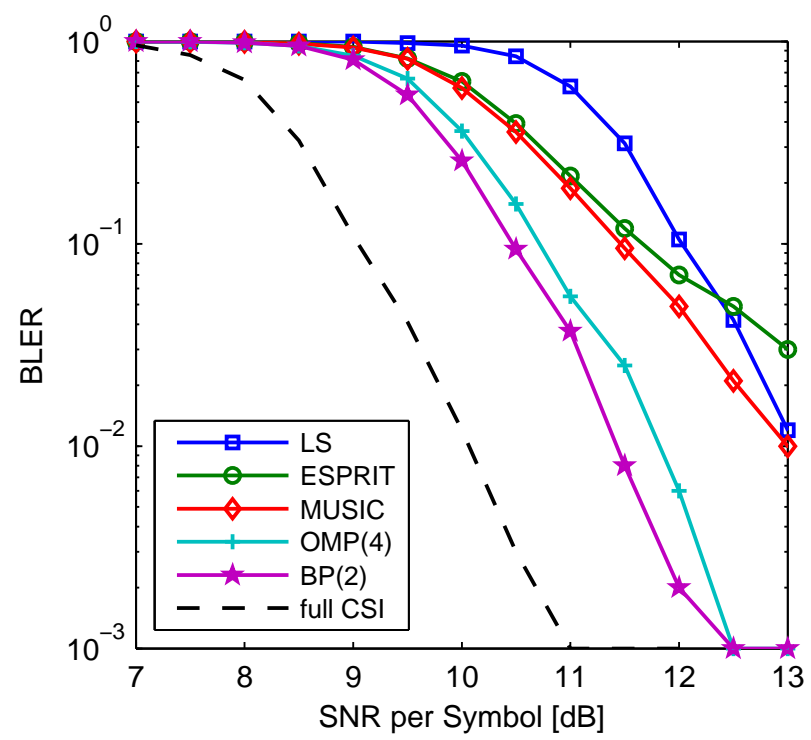

Fig. 3. On less sparse channels $\left(N_{p}=45\right)$, the compressed sensing methods slowly converge to the LS performance, while the subspace based estimators cannot model the composite effect of many irresolvable paths, performing worse than the LS estimator at high SNR.

the equivalent baseband model. In UWA, this is often termed "diffuse" multipath.

To verify this hypothesis, we run the same simulation with a denser channel model. We increase the number of paths to $N_{p}=45$, while keeping the total delay spread constant, leading to closer spaced arrivals. The simulation results in Fig. 3 support our hypothesis, as while all sparse estimators gain less over the LS approach, the subspace methods suffer considerably more.

\section{ICI EFFECTS IN DOPPLER SPREAD CHANNELS}

We now consider the effect of Doppler spread on the system performance. First, we will generate data corresponding to a low degree of Doppler spread and continue using the receiver previously used on the linear time invariant channels, see Section V (also used in [34]-[36]). This reflects well the conditions in UWA communication on days of calm sea, as there will always be a certain degree of Doppler spreading present, even when assumed negligible. As Doppler effects are not addressed, any ICI is treated as additional additive noise, therefore the receiver operates in an ICI-ignorant fashion. We will afterwards proceed to more severe Doppler spread channels, which can only be handled by directly addressing the ICI.

\section{A. ICI-Ignorant Receiver}

To simulate Doppler spread using the path-based channel model, each path is assigned a Doppler rate drawn from a zero mean uniform distribution (we use again $N_{p}=15$ ). With the velocity standard deviation $\sigma_{v}$, the maximum possible Doppler is $\sqrt{3} \sigma_{v} f_{c} / c$ (the sound speed is set to $c=1500 \mathrm{~ms}$ ). We choose a zero-mean Doppler distribution, because a non-zero mean could be removed through the resampling operation.

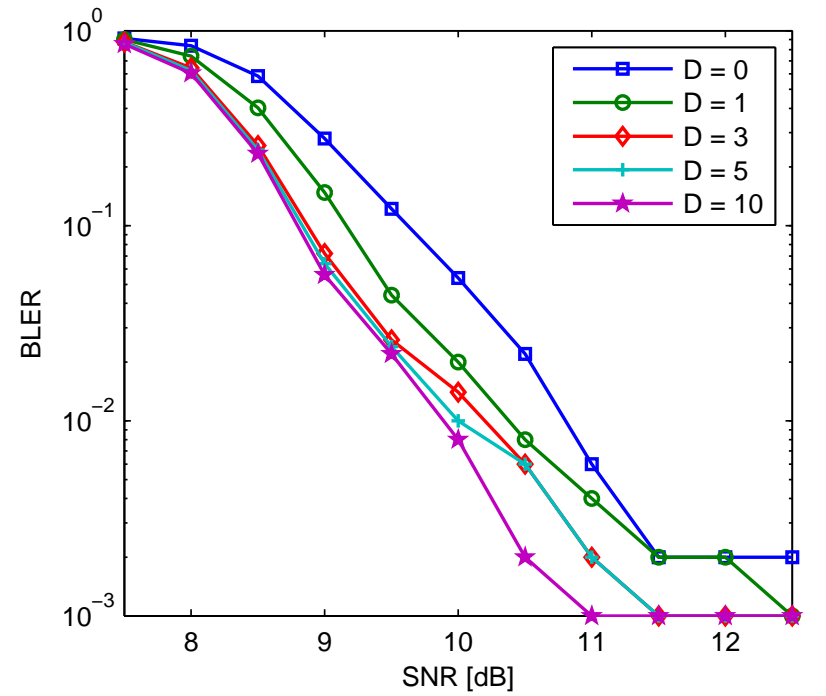

Fig. 4. Perfect channel knowledge, but only $D$ off-diagonals from each side are kept in the channel matrix for data demodulation. The channel has a mild Doppler spread, i.e, the Doppler rates of the simulated path-based model are generated using a uniform distribution with $\sigma_{v}=0.1 \mathrm{~m} / \mathrm{s}$.

1) Equalizer Trade-Off for Mild Doppler Spread: To assess the need for equalization to suppress ICI, we first assume that the receiver has perfect knowledge of all path amplitudes, delays, and Doppler rates. However, the channel mixing matrix $\mathbf{H}$ in (16) will be approximated with a banded structure keeping $D$ off-diagonals to each side (i.e., a total of $2 D+1$ diagonals are retained). We then suppress ICI by using an MMSE equalizer, see (35). This is a trade-off in the sense that by choosing a larger $D$ we can remove more ICI, but will have to accept higher computational complexity in the associated matrix inversion.

Fig. 4 shows the performance for different $D$, where the channel has mild Doppler spread with $\sigma_{v}=0.1 \mathrm{~m} / \mathrm{s}$. We observe that what corresponds to the ICI-ignorant receiver $(D=0)$ works well, being about $1.5 \mathrm{~dB}$ away from the full matrix case. Most of the ICI can be captured by a banded matrix approximation with $D=3$; for $D=10$ the ICI is practically removed and the performance matches closely the full CSI curve for Doppler free channels, see e.g. Fig. 3.

2) Effect of Mild Doppler Spread on Channel Estimation: In Fig. 5, we compare the ICI-ignorant receivers $(D=0)$. That means the channels are estimated the same as on the Doppler free channels in the previous sections, and no ICI is equalized. We find that all receivers can still achieve a low BLER, but at different levels of SNR. This implies that the level of ICI is below the necessary SNR for the LDPC code to decode successfully. The loss in performance is about $1.5 \mathrm{~dB}$ compared to the ICI-free case in Fig. 2. We posit that the performance loss is due to the unaddressed ICI, but that channel estimation is not significantly affected by the model mismatch of the linear time invariant channel assumption. Between the sparse channel estimators, the compressed sensing based algorithms still outperform the subspace algorithms, but less so than on the Doppler free channel. 


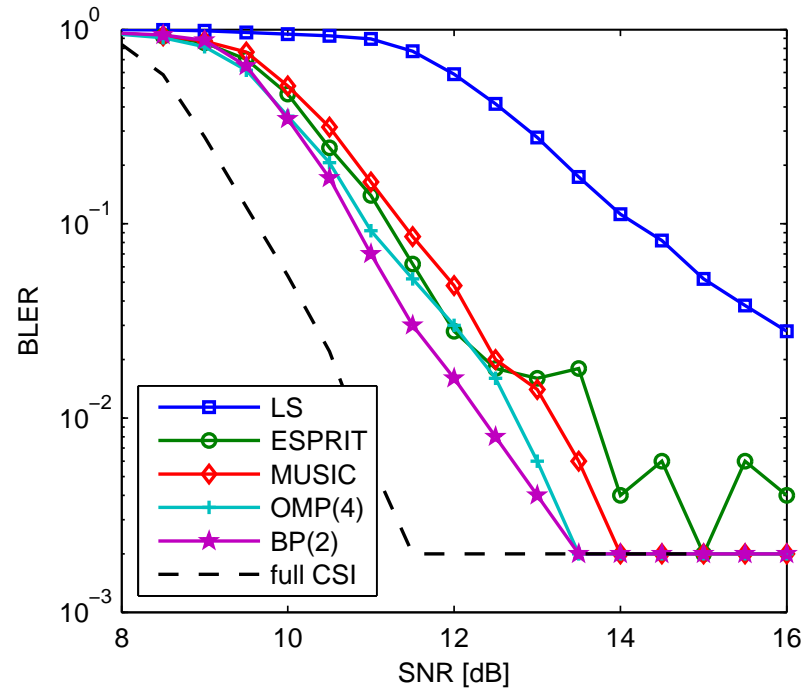

Fig. 5. Performance comparisons for ICI-ignorant receivers with different channel estimation methods; channel has mild Doppler spread $\left(\sigma_{v}=0.1 \mathrm{~m} / \mathrm{s}\right)$.

\section{B. ICI-Aware Receiver}

We now consider channels with more severe Doppler spreads. To improve the channel estimation performance in the presence of severe ICI, we convert 96 data subcarriers into additional pilots by assuming that 96 data symbols are known a priori. The additional pilots are grouped in clusters between zero subcarriers and existing pilots, creating groups of five consecutive known subcarriers. Adjacent observations are needed as to effectively estimate the Doppler rate $b_{p}$ of each path by observing the $\mathrm{ICI}^{2}$.

Since 96 coded symbols are assumed known while the same LDPC code structure is used (code truncation), this leads to an equivalent coding rate of $(336-96) /(672-96) \approx 0.4$. With 16-QAM constellation, the spectral efficiency and the data rate are

$$
\begin{aligned}
\alpha & =\frac{T}{T+T_{g}} \cdot \frac{336-96}{1024} \cdot \log _{2} 16=0.76 \mathrm{bits} / \mathrm{s} / \mathrm{Hz}, \\
R & =\alpha B=7.4 \mathrm{~kb} / \mathrm{s} .
\end{aligned}
$$

1) Equalizer Trade-Off for Severe Doppler Spread: We first assume that the channel is known to assess the need for equalization. The numerical simulation results are depicted in Fig. 6, where $\sigma_{v}=0.25 \mathrm{~m} / \mathrm{s}$. Clearly, ICI-ignorant receivers $(D=0)$ will have very poor performance, which indicates the need for ICI-aware receivers. This means in turn that the ICI needs to be estimated as part of channel estimation, so that equalization can be performed. We also notice that in the full CSI case, once we remove sufficient levels of ICI the performance is about $1 \mathrm{~dB}$ better than in Fig. 4, due to the change in coding rate.

2) Channel Estimation for Severe Doppler Spread Channels: The channels with significant Doppler spread can only be handled by the compressed sensing based estimators. In

\footnotetext{
${ }^{2}$ Optimal pilot placement is still a topic of ongoing reseach. Comparing with random pilot placement as in the compressed sensing research is of interest.
}

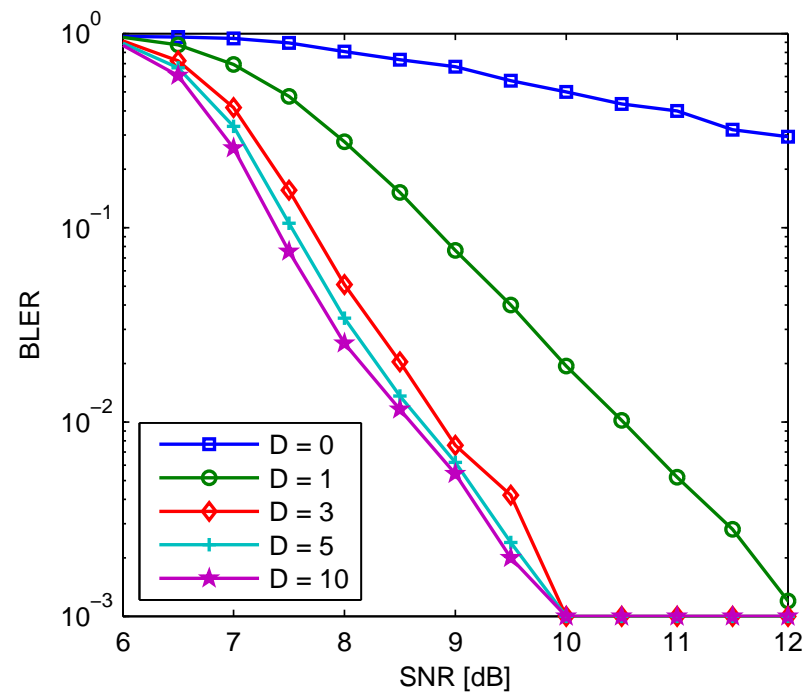

Fig. 6. Perfect channel knowledge, but only $D$ off-diagonals from each side are kept in the channel matrix for data demodulation. The simulated channel has a severe Doppler spread with $\sigma_{v}=0.25 \mathrm{~m} / \mathrm{s}$.

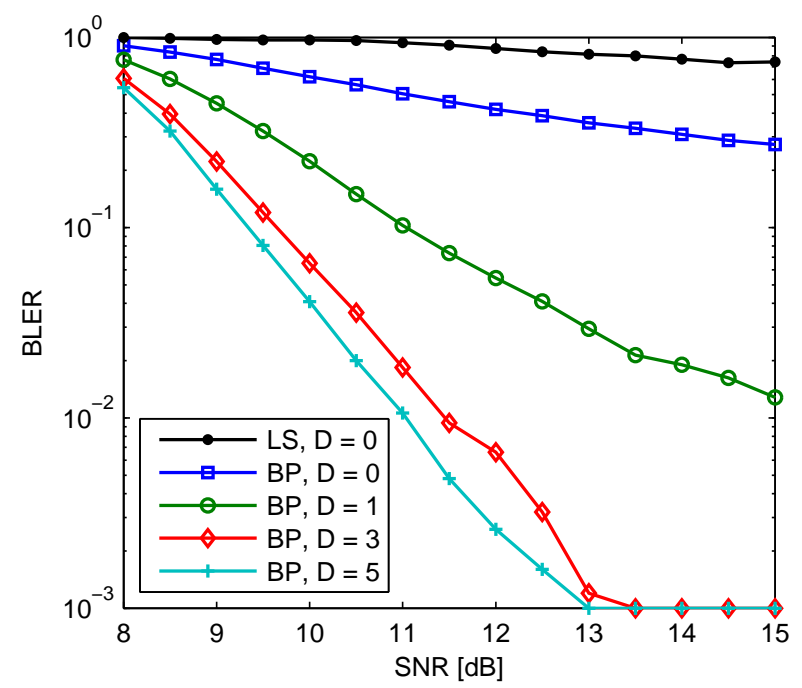

Fig. 7. Performance of ICI-aware BP receiver, where the channel mixing matrix is assumed to have $D$ off diagonals from each side; compared to full CSI the performance degrades by about $3 \mathrm{~dB}$.

addition to delay, we introduce dictionaries that also consider fifteen different Doppler rates uniformly distributed within $\left[-b_{\max }, b_{\max }\right]$, where $b_{\max }=v_{\max } / c=5 \cdot 10^{-4}$. Although both BP and OMP can handle ICI-aware channel estimation, we here focus only on $\mathrm{BP}^{3}$. As comparison we include the LS and the BP algorithms that assume no Doppler as previously $(D=0)$, but benefit from the increased number of pilots ${ }^{4}$. Simulation results are in Fig. 7. We observe that performance significantly improves by considering ICI explicitly through the increase of $D$. The trend with increasing $D$ is similar as in Fig. 6, but generally the performance is about $3 \mathrm{~dB}$ worse

\footnotetext{
${ }^{3}$ In previous work we found that BP always outperforms OMP, especially in the ICI-aware setting [45].

${ }^{4}$ The same is not possible for the subspace algorithms, as the pilot pattern no longer has the shift invariance property.
} 

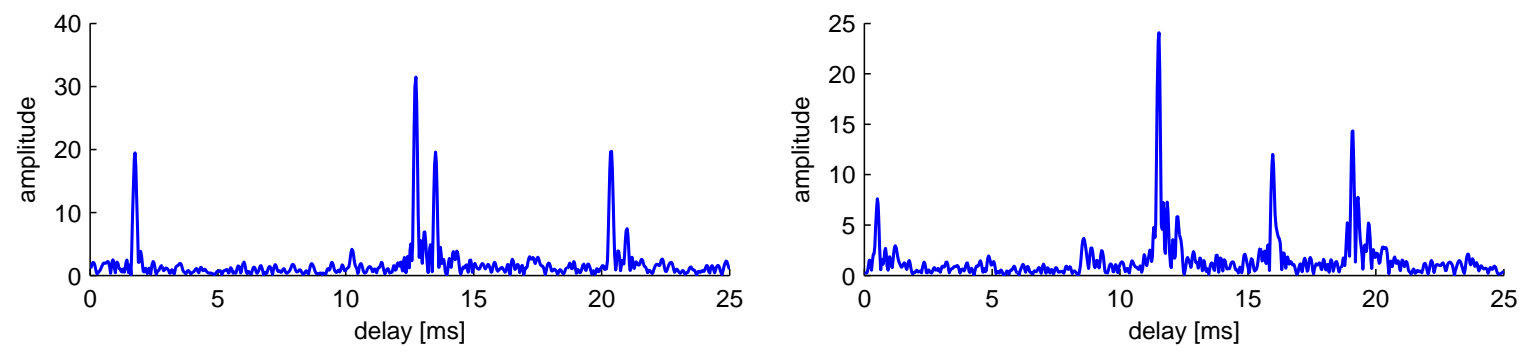

Fig. 8. Two example channels from the GLINT'08 experiment; the impulse response is sparse and has a delay spread of about $20 \mathrm{~ms}$.

TABLE II

PARAMETERS OF ZP-OFDM IN GLINT'08 EXPERIMENT.

\begin{tabular}{|l|c|c|}
\hline carrier frequency & $f_{c}$ & $25 \mathrm{kHz}$ \\
bandwidth & $B$ & $7.8125 \mathrm{kHz}$ \\
no. subcarriers & $K$ & 1024 \\
symbol duration & $T$ & $131.072 \mathrm{~ms}$ \\
subcarrier spacing & $\Delta f:=1 / T$ & $7.63 \mathrm{~Hz}$ \\
guard interval & $T_{g}$ & $25 \mathrm{~ms}$ \\
\hline
\end{tabular}

due to imperfect channel estimation.

\section{EXPERIMENTAL RESUlTS}

As numerical simulation can only capture some of the effects of real UWA communication, we next use data experimentally recorded in two different environments: i) during the GLINT'08 experiment; and ii) during the SPACE'08 experiment. We will start with the GLINT'08 experiment as it corresponds more so to the mild Doppler spread scenario, then proceed to the SPACE'08 experiment, as it included stormy days with strong wind and wave activity leading to what we call severe Doppler spread.

\section{A. ICI-Ignorant Receivers for GLINT'08 Experiment}

The first data we consider was recorded during the GLINT'08 experiment, in the area around Pianosa, just south of Elba, off the coast of Italy, in July 2008. At this point of the Mediterranean, the water depth is about $90 \mathrm{~m}$, and the data was recorded by a hydrophone array with four elements. We will focus on data recorded on three days of the experiment, July 25 to July 27 of 2008.

Although the general OFDM structure is the same as in Section V, i.e., total number of subcarriers, split into data, pilots, and zeros, the carrier frequency, bandwidth, and symbol duration are different, as specified in Table II. With this the spectral efficiency for 16-QAM is the same, but the data rate is slightly less, due to the smaller bandwidth:

$$
\begin{aligned}
\alpha & =\frac{T}{T+T_{g}} \cdot \frac{672}{1024} \cdot \frac{1}{2} \cdot \log _{2} 16=1.1 \mathrm{bits} / \mathrm{s} / \mathrm{Hz}, \\
R & =\alpha B=8.6 \mathrm{~kb} / \mathrm{s} .
\end{aligned}
$$

We will additionally consider 64-QAM for increased data rate:

$$
\begin{aligned}
\alpha & =\frac{T}{T+T_{g}} \cdot \frac{672}{1024} \cdot \frac{1}{2} \cdot \log _{2} 64=1.65 \mathrm{bits} / \mathrm{s} / \mathrm{Hz}, \\
R & =\alpha B=12.96 \mathrm{~kb} / \mathrm{s} .
\end{aligned}
$$

Two recorded channel impulse responses are plotted in Fig. 8; we notice that the channels are extremely sparse, with about four noticeable clusters, and feature a total delay spread of about $20 \mathrm{~ms}$. The data from the three days was recorded under the following conditions,

- July 25: Recorded at a distance of $905 \mathrm{~m}$, drift negligible.

- July 26: Recorded at a distance of $1,720 \mathrm{~m}$, drifting at 0.7 knots $(0.36 \mathrm{~m} / \mathrm{s})$.

- July 27: Recorded at a distance of $1,500 \mathrm{~m}$, drifting at 0.6 knots $(0.31 \mathrm{~m} / \mathrm{s})$.

For each day, we use five recorded files, for each file 15 OFDM blocks are transmitted, leading to a total of 75 transmitted blocks to assess the BLER.

Inspecting the performance results in Fig. 9, we notice that almost all blocks can be decoded correctly, for both 16-QAM and 64-QAM. Generally BP is the best, followed by OMP; the subspace methods can be better or worse than the LS estimator at times. The overall good performance makes differentiation difficult. The transmitter motion seems to be well compensated by the resampling and fine Doppler shift compensation, as it does not degrade the performance. We conclude that the calm water surface during the experiment does not lead to noticeably Doppler spread channels.

\section{B. ICI-Ignorant Receivers for SPACE'08 Experiment}

The SPACE'08 experiment was held off the coast of Martha's Vineyard, MA, from Oct. 14 to Nov. 1, 2008. The water depth was about 15 meters. We consider three receivers, labeled as S1, S3, and S5, which were $60 \mathrm{~m}, 200 \mathrm{~m}$, and $1,000 \mathrm{~m}$ from the transmitter, respectively. Each receiver array has at least twelve hydrophones. We plot the performance combining an increasing number of phones to increase the effective SNR and show performance differences. We consider recorded data from two different days, Julian Dates 297 and 300 , where one day has rather calm sea and one day has severe wind activity, respectively. For each day, there are twelve recorded files consisting of twenty OFDM symbols each. On the second day, the five files recorded during the afternoon were severely distorted and therefore unusable; we focus on the remaining seven files recorded during the morning and evening.

The OFDM parameters are identical to those in Sec. VI-A, given in Table I; hence, the achieved spectral efficiency and the data rate are in (36) and (37), respectively. 


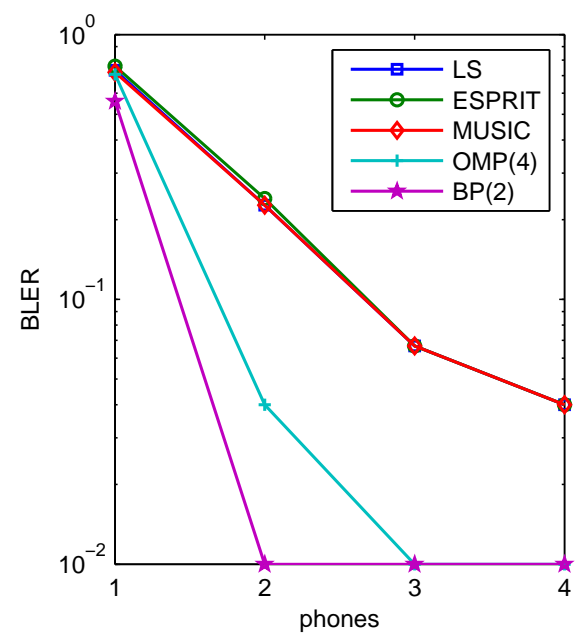

(a) July 25, 16-QAM

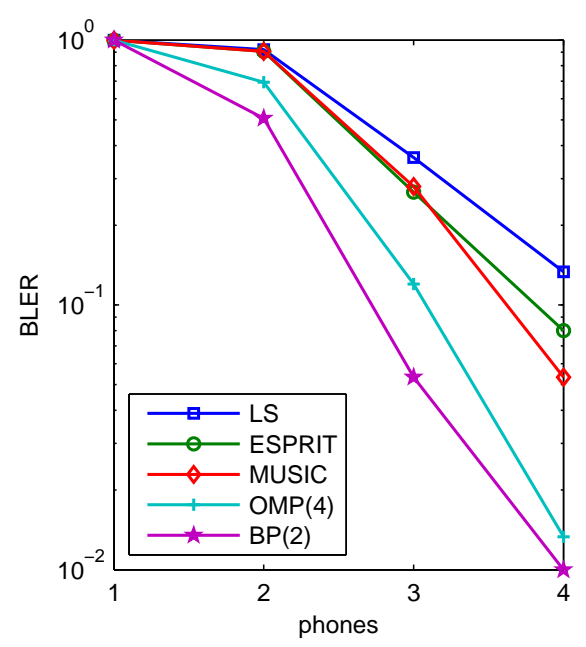

(d) July 25, 64-QAM

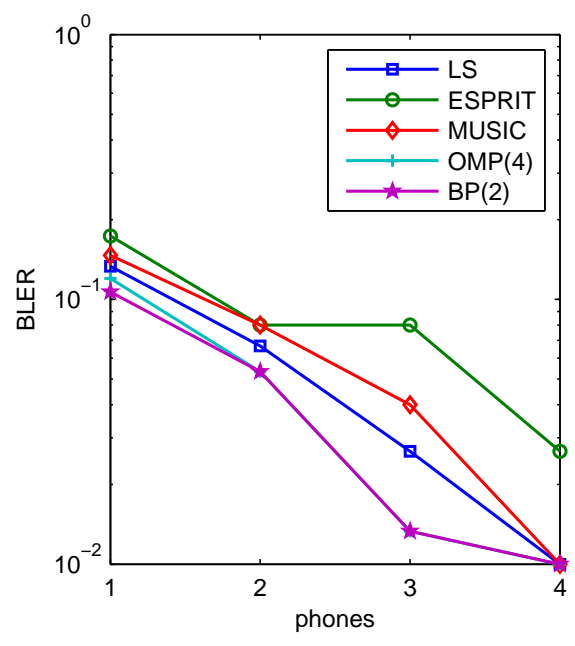

(b) July 26, 16-QAM

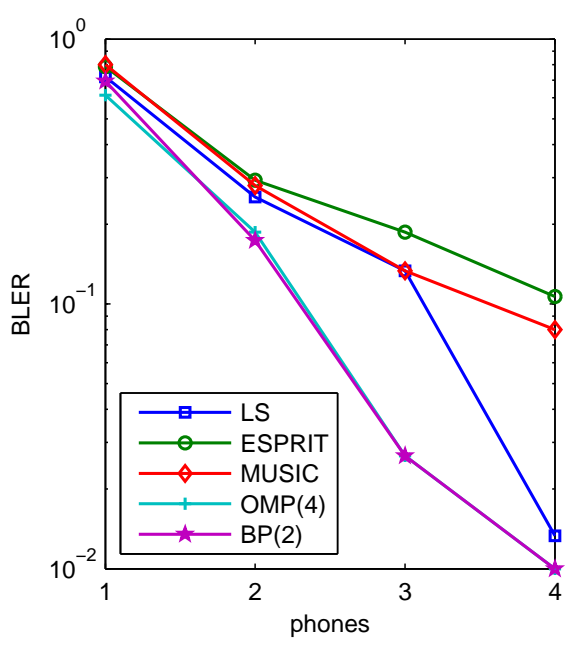

(e) July 26, 64-QAM

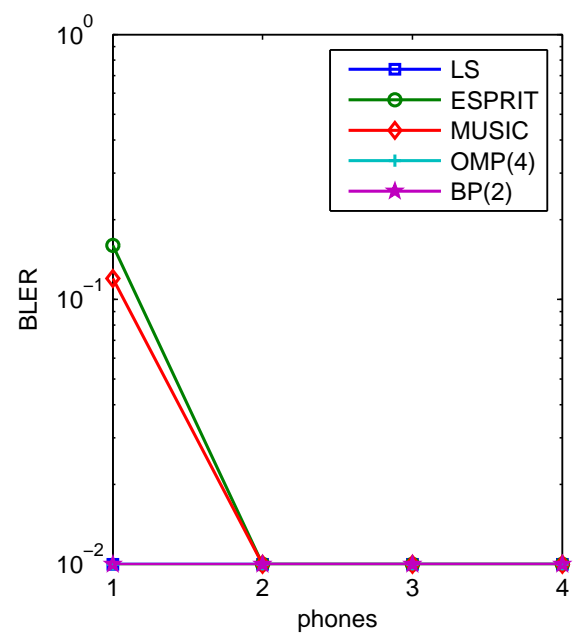

(c) July 27, 16-QAM

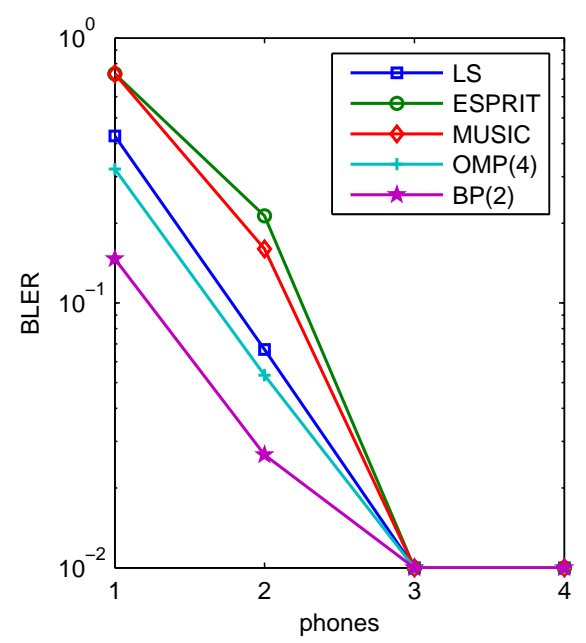

(f) July 27, 64-QAM

Fig. 9. Performance results from the GLINT experiment using ICI-ignorant receivers for two data rates, recorded over three days.

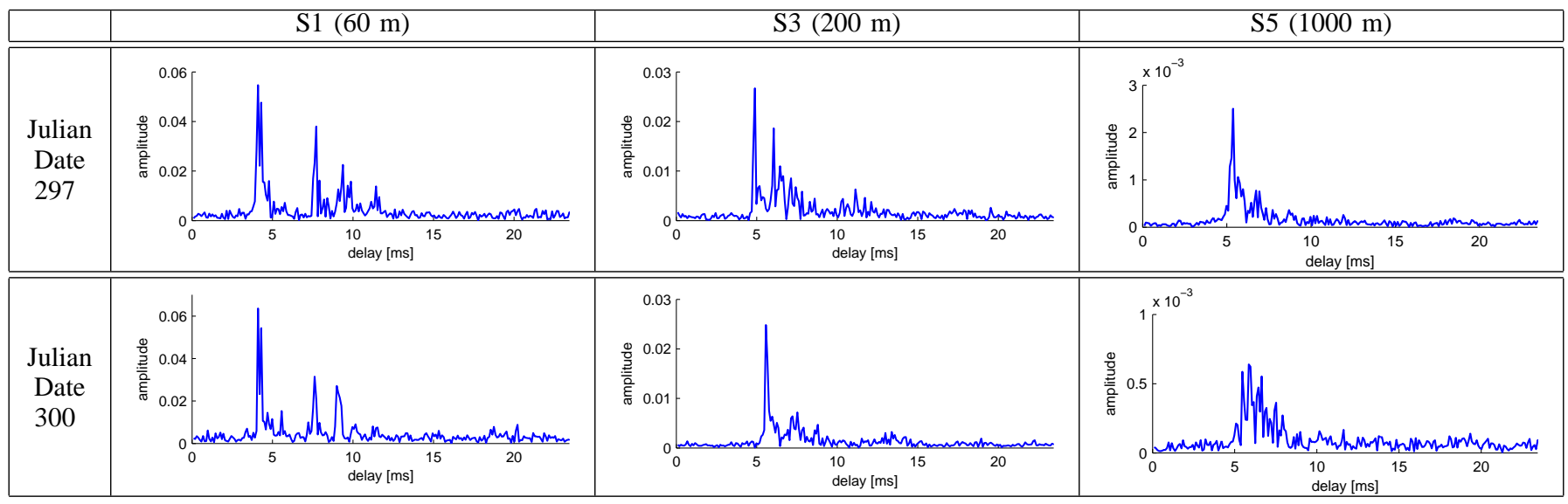

Fig. 10. Examples of channel responses from the SPACE'08 experiment, taken from the LS estimate. 


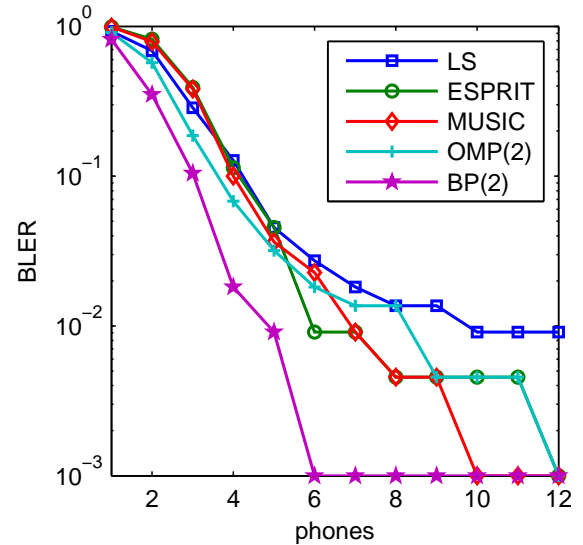

(a) Julian Date 297, S1

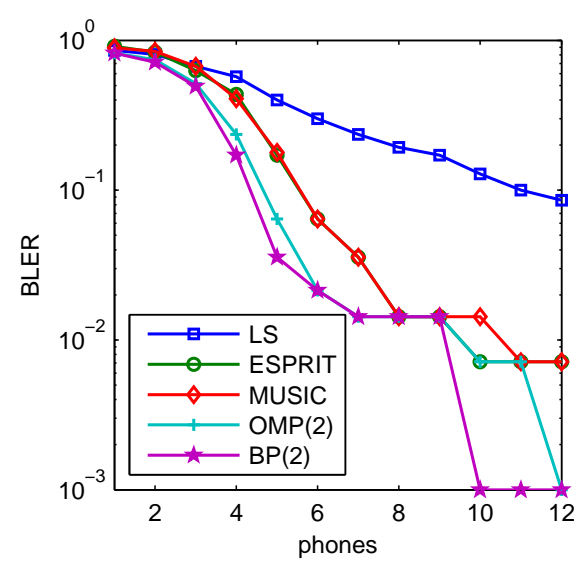

(d) Julian Date 300, S1

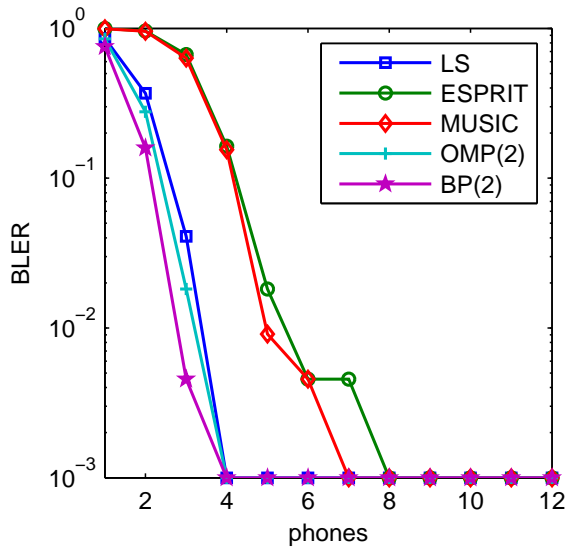

(b) Julian Date 297, S3

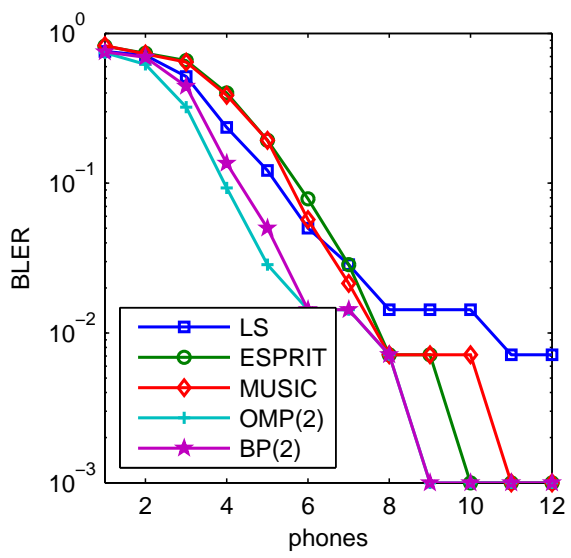

(e) Julian Date 300, S3

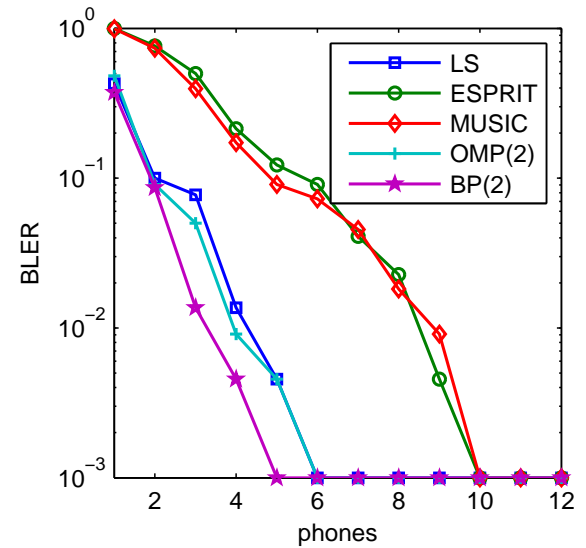

(c) Julian Date 297, S5

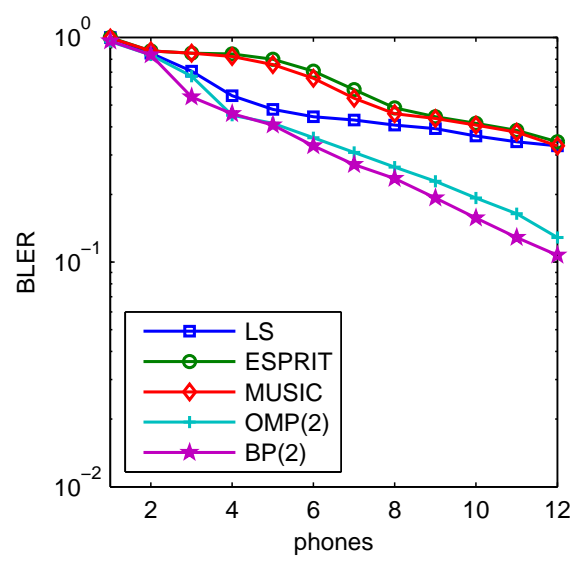

(f) Julian Date 300, S5

Fig. 11. Performance results from the SPACE'08 experiment using ICI-ignorant receivers at three different locations (S1, S3, and S5), first on a day with calm weather, then a day with stormy conditions.

In this subsection, we test ICI-ignorant receivers. The sample channel responses based on the LS estimators at different receiver locations are shown in Fig. 10.

1) S1 Data $(60 \mathrm{~m})$ : At a short distance of only $60 \mathrm{~m}$ and considering the shallow water depth, we expect rich multipath and significant Doppler variation due to the geometry. This makes this receiver the most challenging in terms of its channel response, but the easiest in terms of received signal strength or SNR. From Fig. 10, we notice that there are three to four significant clusters of similar strength. The total delay spread is around $10 \mathrm{~ms}$.

In Fig. 11 we see the BLER performance for Julian Dates 297 and 300. As in the numerical simulation the order of compressed sensing, subspace, LS stays the same.

2) S3 Data $(200 \mathrm{~m})$ : The middle distance might be the best tradeoff between channel difficulty and received SNR. The example channel responses in Fig. 10 seem to be more contained, with a more dominating first cluster. The BLER performance in Fig. 11 is generally better compared to the S1 receiver, where the LS performance gains relative to the sparse estimators, outperforming the subspace methods on Julian date 297 and coming quite close on Julian date 300.
3) S5 Data $(1000 \mathrm{~m})$ : At the $1 \mathrm{~km}$ distance only one significant cluster can be spotted in the channel estimates, and at the stormy day (Julian Date 300) the received energy seems to be vanishingly small, c.f. Fig. 10. Accordingly the trend of the LS channel estimator closing in on the compressed sensing algorithms continues, with the subspace methods not able to handle this diffuse multipath. On the stormy day the performance is generally not as good, with even the CS algorithms successfully recovering only about $80 \%$ of the OFDM blocks.

\section{ICI-Aware Receivers for SPACE'08 Experiment}

We saw that on the stormy day (Julian Date 300), the performance was limited, most likely due to ICI caused by significant Doppler spread that degrades the effective SNR of the ICI-ignorant receivers. We now focus on Julian Date 300 to test the effectiveness of ICI-aware receivers based on BP.

The OFDM parameters are identical to those in Section VI-B, given in Table I, but we will also consider 64-QAM; hence, the achieved spectral efficiency and the data rate for 16QAM are in (38) and (39), respectively, while for 64-QAM 


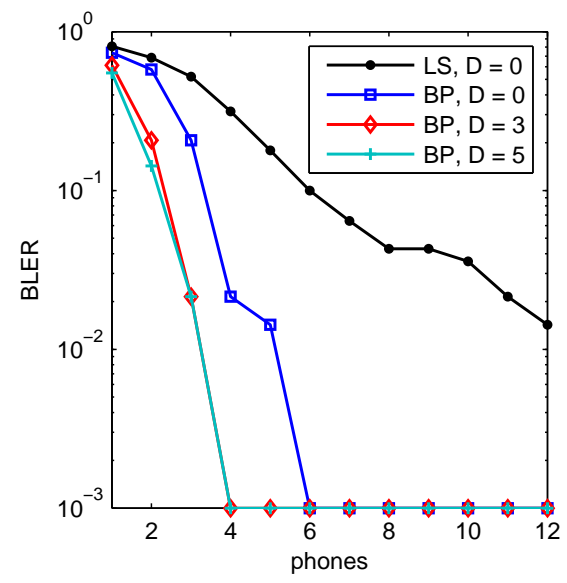

(a) 16-QAM, S1

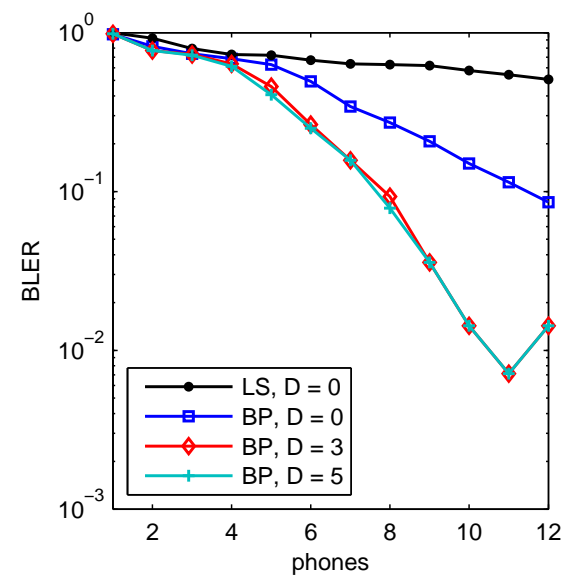

(d) 64-QAM, S1

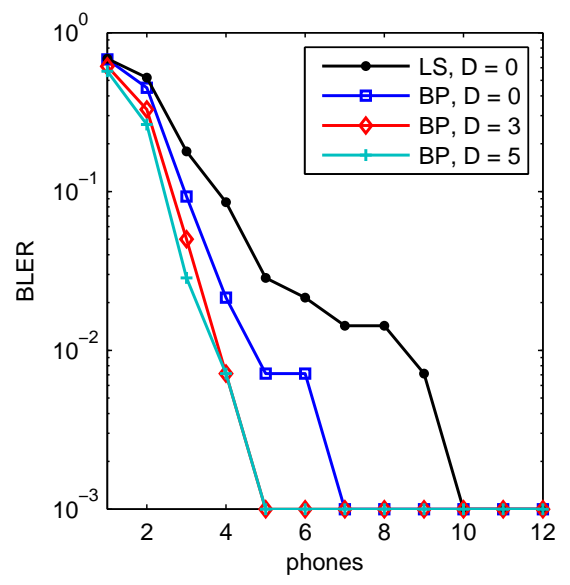

(b) 16-QAM, S3

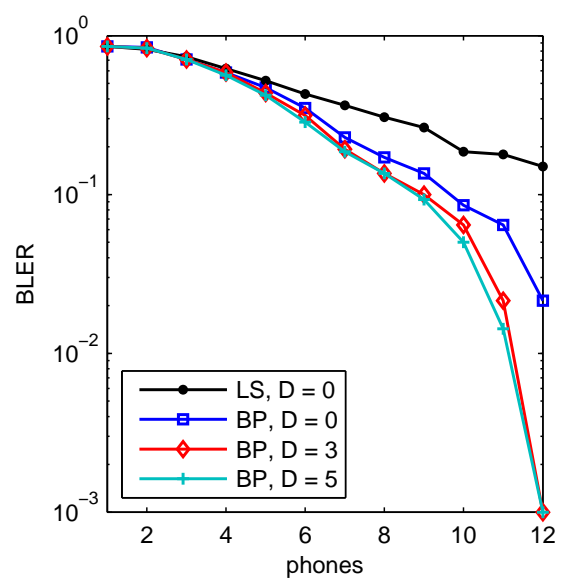

(e) 64-QAM, S3

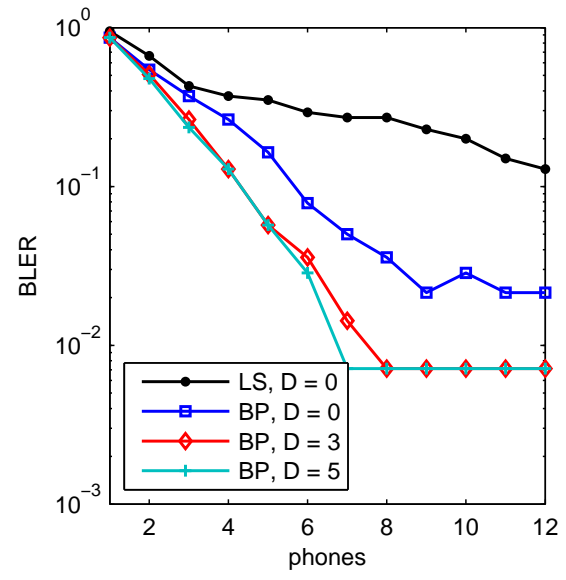

(c) 16-QAM, S5

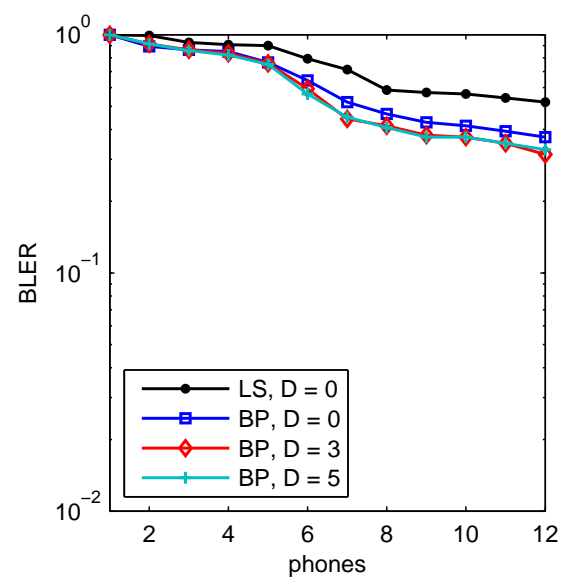

(f) 64-QAM, S5

Fig. 12. Performance results from the SPACE'08 experiment using the ICI-aware BP receiver; we focus on Julian date 300 and consider both 16-QAM (data rate of $7.4 \mathrm{~kb} / \mathrm{s}$ ) and 64-QAM (data rate of $11.1 \mathrm{~kb} / \mathrm{s}$ ).

we have:

$$
\begin{aligned}
\alpha & =\frac{T}{T+T_{g}} \cdot \frac{336-96}{1024} \cdot \log _{2} 64=1.14 \mathrm{bits} / \mathrm{s} / \mathrm{Hz}, \\
R & =\alpha B=11.1 \mathrm{~kb} / \mathrm{s} .
\end{aligned}
$$

The performance improvement for ICI-aware receivers can be seen in Fig. 12. As a comparison we also include LS and BP channel estimators operating ICI-ignorant $(D=0)$, as they also benefit from the additional pilots and reduced coding rate.

These plots clearly highlight again that using sparse channel estimation a significant performance gain can be realized compared to a conventional LS channel estimator (difference between LS and BP for $D=0$ ). Furthermore, on channels with severe Doppler spread adopting ICI-aware channel estimation and equalization another significant gain in performance can be achieved.

\section{CONCLUSION}

We considered sparse channel estimation for multicarrier underwater acoustic communication. Based on the path-based channel model, we linked well-known subspace methods from the array-processing literature to the channel estimation problem. Also we employed recent compressed sensing methods, namely Orthogonal Matching Pursuit (OMP) and Basis Pursuit (BP). Based on the continuous time characterization of the path delays, we suggested the use of finer delay resolution overcomplete dictionaries. We also extended the compressed sensing receivers to handle channels with different Doppler scales on different paths, supplying intercarrier interference (ICI) pattern estimates that can be used to equalize the ICI. Using extensive numerical simulation and experimental results, we find that in comparison to the LS receiver the subspace methods show significant performance increase on channels that are sparse, but perform worse if most received energy comes from diffuse multipath. The compressed sensing algorithms do not suffer this drawback, and benefit significantly from the increased time resolution using overcomplete dictionaries. When accounting for different Doppler scales on different paths, BP can effectively handle channels with very large Doppler spread. 


\section{REFERENCES}

[1] I. F. Akyildiz, D. Pompili, and T. Melodia, "Challenges for efficient communication in underwater acoustic sensor networks," ACM SIGBED Review, vol. 1, no. 1, pp. 3-8, Jul. 2004.

[2] J.-H. Cui, J. Kong, M. Gerla, and S. Zhou, "The challenges of building mobile underwater wireless networks for aquatic applications," IEEE Network, Special Issue on Wireless Sensor Networking, vol. 20, no. 3, pp. 12-18, May/June 2006.

[3] J. Heidemann, U. Mitra, J. C. Preisig, M. Stojanovic, and M. Zorzi, "Guest editorial - underwater wireless communication networks," IEEE J. Select. Areas Commun., vol. 26, no. 9, pp. 1617-1619, Dec. 2008.

[4] A. C. Singer, J. K. Nelson, and S. S. Kozat, "Signal processing for underwater acoustic communications," IEEE Communications Magazine, vol. 47, no. 1, pp. 90-96, Jan. 2009.

[5] T. H. Eggen, A. B. Baggeroer, and J. C. Preisig, "Communication over Doppler spread channels. Part I: Channel and receiver presentation," IEEE J. Ocean. Eng., vol. 25, no. 1, pp. 62-71, Jan. 2000.

[6] D. B. Kilfoyle and A. B. Baggeroer, "The state of the art in underwater acoustic telemetry," IEEE J. Ocean. Eng., vol. 25, no. 1, pp. 4-27, Jan. 2000.

[7] F. Qu and L. Yang, "Basis expansion model for underwater acoustic channels?" in Proc. of MTS/IEEE OCEANS Conf., Quèbec City, Quèbec, Sep. 2008.

[8] S.-J. Hwang and P. Schniter, "Efficient multicarrier communication for highly spread underwater acoustic channels," IEEE J. Select. Areas Commun., vol. 26, no. 9, pp. 1674-1683, Dec. 2008.

[9] G. Leus and P. A. van Walree, "Multiband OFDM for covert acoustic communications," IEEE J. Select. Areas Commun., vol. 26, no. 9, pp. 1662-1673, Dec. 2008.

[10] W. Li and J. C. Preisig, "Estimation of rapidly time-varying sparse channels," IEEE J. Ocean. Eng., vol. 32, no. 4, pp. 927-939, Oct. 2007.

[11] M. Stojanovic, "OFDM for underwater acoustic communications: Adaptive synchronization and sparse channel estimation," in Proc. of Intl. Conf. on Acoustics, Speech and Signal Proc., Las Vegas, NV, Apr. 2008.

[12] C.-J. Wu and D. W. Lin, "Sparse channel estimation for OFDM transmission based on representative subspace fitting," in Proc. of Vehicular Technology Conf., Stockholm, Sweden, May 2005.

[13] M. R. Raghavendra and K. Giridhar, "Improving channel estimation in OFDM systems for sparse multipath channels," IEEE Signal Processing Lett., vol. 12, no. 1, pp. 52-55, Jan. 2005.

[14] C. Carbonelli, S. Vedantam, and U. Mitra, "Sparse channel estimation with zero tap detection," IEEE Trans. Wireless Commun., vol. 6, no. 5, pp. 1743-1763, May 2007.

[15] O. Rabaste and T. Chonavel, "Estimation of multipath channels with long impulse response at low SNR via an MCMC method," IEEE Trans. Signal Processing, vol. 55, no. 4, pp. 1312-1325, Apr. 2007.

[16] E. Candes, J. Romberg, and T. Tao, "Robust uncertainty principles: Exact signal reconstruction from highly incomplete frequency information," IEEE Trans. Inform. Theory, vol. 52, no. 2, pp. 489-509, Feb. 2006.

[17] D. Donoho, "Compressed sensing," IEEE Trans. Inform. Theory, vol. 52, no. 4, pp. 1289-1306, Apr. 2006

[18] E. Candes and T. Tao, "Near-optimal signal recovery from random projections: Universal encoding strategies?" IEEE Trans. Inform. Theory, vol. 52, no. 12, pp. 5406-5425, Dec. 2006.

[19] R. Baraniuk, "Compressive sensing," IEEE Signal Processing Magazine, vol. 24, no. 4, pp. 118-121, Jul. 2007.

[20] S. F. Cotter and B. D. Rao, "Sparse channel estimation via matching pursuit with application to equalization," IEEE Trans. Commun., vol. 50, no. 3, pp. 374 - 377, Mar. 2002.

[21] G. Z. Karabulut and A. Yongacoglu, "Sparse channel estimation using orthogonal matching pursuit algorithm," in Proc. of Vehicular Technology Conf., Los Angeles, CA, Sep. 2004.

[22] C.-J. Wu and D. W. Lin, "A group matching pursuit algorithm for sparse channel estimation for OFDM transmission," in Proc. of Intl. Conf. on Acoustics, Speech and Signal Proc., Toulouse, France, May 2006.

[23] C. Carbonelli and U. Mitra, "A simple sparse channel estimator for underwater acoustic channels," in Proc. of MTS/IEEE OCEANS Conf., Vancouver, Canada, Oct. 2007.

[24] J. L. Paredes, G. R. Arce, and Z. Wang, "Ultra-wideband compressed sensing: Channel estimation," IEEE J. Select. Topics Signal Proc., vol. 1, no. 3, pp. 383-395, Oct. 2007.

[25] B. Friedlander, "Random projections for sparse channel estimation and equalization," in Proc. of Asilomar Conf. on Signals, Systems, and Computers, Pacific Grove, CA, Oct. 2006.
[26] W. U. Bajwa, J. Haupt, G. Raz, and R. Nowak, "Compressed channel sensing," in Proc. of Conf. on Information Sciences and Systems (CISS), Princeton, NJ, Mar. 2008.

[27] M. Sharp and A. Scaglione, "Application of sparse signal revocery to pilot-assisted channel estimation," in Proc. of Intl. Conf. on Acoustics, Speech and Signal Proc., Las Vegas, NV, Apr. 2008.

[28] G. Taubock and F. Hlawatsch, "A compressed sensing technique for OFDM channel estimation in mobile environments: Exploiting channel sparsity for reducing pilots," in Proc. of Intl. Conf. on Acoustics, Speech and Signal Proc., Las Vegas, NV, Apr. 2008.

[29] _ "Compressed sensing based estimation of doubly selective channels using a sparsity-optimized basis expansion," in Proc. of European Signal Processing Conf. (EUSIPCO), Lausanne, Switzerland, Aug. 2008

[30] W. U. Bajwa, A. Sayeed, and R. Nowak, "Learning sparse doublyselective channels," in Proc. of Allerton Conf. on Communications, Control and Computing, Sep. 2008, pp. 575-582.

[31] — - "Compressed sensing of wireless channels in time, frequency, and space," in Proc. of Asilomar Conf. on Signals, Systems, and Computers, Pacific Grove, CA, Oct. 2008.

[32] R. A. Iltis, "Iterative joint decoding and sparse channel estimation for single-carrier modulation," in Proc. of Intl. Conf. on Acoustics, Speech and Signal Proc., Las Vegas, NV, Apr. 2008, pp. 2689-2692.

[33] T. Kang and R. A. Iltis, "Iterative carrier frequency offset and channel estimation for underwater acoustic OFDM systems," IEEE J. Select. Areas Commun., vol. 26, no. 9, pp. 1650-1661, Dec. 2008.

[34] B. Li, S. Zhou, M. Stojanovic, L. Freitag, and P. Willett, "Multicarrier communication over underwater acoustic channels with nonuniform Doppler shifts," IEEE J. Ocean. Eng., vol. 33, no. 2, pp. 198-209, Apr. 2008.

[35] S. Mason, C. R. Berger, S. Zhou, and P. Willett, "Detection, synchronization, and Doppler scale estimation with multicarrier waveforms in underwater acoustic communication," IEEE J. Select. Areas Commun., vol. 26, no. 9, pp. 1638-1649, Dec. 2008.

[36] J. Huang, S. Zhou, and P. Willett, "Nonbinary LDPC coding for multicarrier underwater acoustic communication," IEEE J. Select. Areas Commun., vol. 26, no. 9, pp. 1684-1696, Dec. 2008.

[37] H. Van Trees, Optimum Array Processing, 1st ed., ser. Detection, Estimation, and Modulation Theory (Part IV). New York: John Wiley \& Sons, Inc., 2002.

[38] S.-J. Kim, K. Koh, M. Lustig, S. Boyd, and D. Gorinevsky, "An interiorpoint method for large-scale $l_{1}$-regularized least squares," IEEE J. Select. Topics Signal Proc., vol. 1, no. 4, pp. 606-617, Dec. 2007.

[39] M. Stojanovic, "Low complexity OFDM detector for underwater channels," in Proc. of MTS/IEEE OCEANS Conf., Boston, MA, Sept. 18-21, 2006.

[40] T.-J. Shan, M. Wax, and T. Kailath, "On spatial smoothing for directionof-arrival estimation of coherent signals," IEEE Trans. Signal Processing, vol. 33, no. 4, pp. 806-811, Aug. 1985.

[41] M. Pesavento, A. B. Gershman, and M. Haardt, "Unitary root-MUSIC with a real-valued eigendecomposition: A theoretical and experimental performance study," IEEE Trans. Signal Processing, vol. 48, no. 5, pp. 1306-1314, May 2000.

[42] J. A. Tropp and A. C. Gilbert, "Signal recovery from random measurements via orthogonal matching pursuit," IEEE Trans. Inform. Theory, vol. 53, no. 12, pp. 4655-4666, Dec. 2007.

[43] J. G. Proakis, Digital Communications, 4th ed. New York: McGrawHill, 2001.

[44] C. R. Berger, S. Zhou, Y. Wen, K. Pattipati, and P. Willett, "Optimizing joint erasure- and error-correction coding for wireless packet transmissions," IEEE Trans. Wireless Commun., vol. 7, no. 11, pp. 4586-4595, Nov. 2008.

[45] C. R. Berger, S. Zhou, J. Preisig, and P. Willett, "Sparse channel estimation for multicarrier underwater acoustic communication: From subspace methods to compressed sensing," in Proc. of MTS/IEEE OCEANS Conf., Bremen, Germany, May 2009. 


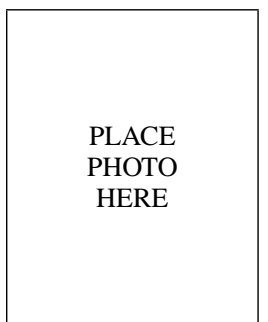

Christian R. Berger (S'05-M'09) was born in Heidelberg, Germany, on September 12, 1979. He received the Dipl.-Ing. degree from the Universität Karlsruhe (TH), Karlsruhe, Germany in 2005, and the Ph.D. degree from the University of Connecticut, Storrs, in 2009, both in electrical engineering.

In the summer of 2006, he was as a visiting scientist at the Sensor Networks and Data Fusion Department of the FGAN Research Institute, Wachtberg, Germany. He is currently a post-doctoral researcher at the Department of Electrical and Computer Engineering, Carnegie Mellon University, Pittsburgh, USA. His research interests lie in the areas of communications and signal processing, including distributed estimation in wireless sensor networks, wireless positioning and synchronization, underwater acoustic communications and networking.

Dr. Berger has served as a reviewer for the IEEE TRANSACTIONS ON SIGNal Processing, IEEE Transactions Wireless Communications, and IEEE TRANSACTIONS AEROSPACESPACE AND ELECTRONIC SYSTEMS. In 2008 he was member of the technical program committee and session chair for the 11th International Conference on Information Fusion in Cologne, Germany.

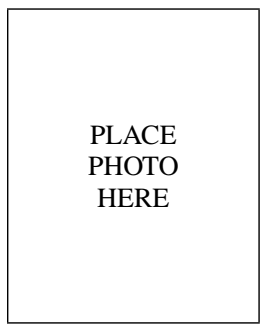

Shengli Zhou (M'03) received the B.S. degree in 1995 and the M.Sc. degree in 1998, from the University of Science and Technology of China (USTC), Hefei, both in electrical engineering and information science. He received his Ph.D. degree in electrical engineering from the University of Minnesota (UMN), Minneapolis, in 2002

$\mathrm{He}$ has been an assistant professor with the Department of Electrical and Computer Engineering at the University of Connecticut (UCONN), Storrs, 2003-2009, and now is an associate professor. He holds a United Technologies Corporation (UTC) Professorship in Engineering Innovation, 2008-2011. His general research interests lie in the areas of wireless communications and signal processing. His recent focus is on underwater acoustic communications and networking.

Dr. Zhou has served as an associate editor for IEEE Transactions on Wireless Communications from Feb. 2005 to Jan. 2007, and is now an associate editor for IEEE Transactions on Signal Processing. He received the 2007 ONR Young Investigator award and the 2007 Presidential Early Career Award for Scientists and Engineers (PECASE).

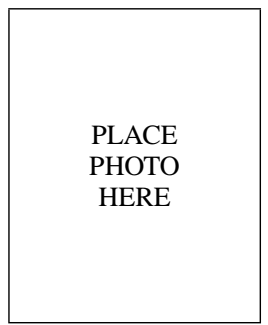

James C. Preisig (S'79-M'80) received the B.S. degree in electrical engineering from the United States Coast Guard Academy, New London, CT, in 1980, the S.M. and E.E. degrees in electrical engineering from the Massachusetts Institute of Technology, Cambridge, in 1988, and the Ph.D. degree in electrical and ocean engineering from the Massachusetts Institute of Technology/Woods Hole Oceanographic Institution (WHOI) Joint Program in Oceanography and Oceanographic Engineering, Cambridge, MA, in 1992.

He was a Postdoctoral Investigator at WHOI from 1992 to 1994 and a Visiting Assistant Professor at Northeastern University, Boston, MA, from 1994 to 1997. Since July 1997, he has been on the scientific staff of the Department of Applied Ocean Physics and Engineering, WHOI, and is currently an Associate Scientist with Tenure. His research interests are in the areas of adaptive signal processing, system identification, underwater acoustic propagation modeling, underwater acoustic communications, and numerical optimization.

Dr. Preisig is the recipient of the 1999 U.S. Office of Naval Research Ocean Acoustics Young Faculty Award and is a member of the Acoustical Society of America's Underwater Acoustics and Signal Processing Technical Committees. He is also an Associate Editor of the IEEE JOURNAL OF OCEANIC ENGINEERING and served as a member of the IEEE Sensor Array and Multichannel Signal Processing Technical Committee from 1998 to 2004 .

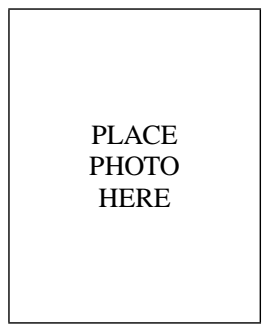

Peter Willett (F'03) received his BASc (Engineering Science) from the University of Toronto in 1982 and his $\mathrm{PhD}$ degree from Princeton University in 1986

He has been a faculty member at the University of Connecticut ever since, and since 1998 has been a Professor. His primary areas of research have been statistical signal processing, detection, machine learning, data fusion and tracking. He has interests in and has published in the areas of change/abnormality detection, optical pattern recognition, communications and industrial/security condition monitoring.

Dr. Willett is editor-in-chief for IEEE Transactions on Aerospace and Electronic Systems, and until recently was associate editor for three active journals: IEEE Transactions on Aerospace and Electronic Systems (for Data Fusion and Target Tracking) and IEEE Transactions on Systems, Man, and Cybernetics, parts A and B. He is also associate editor for the IEEE AES Magazine, editor of the AES Magazines periodic Tutorial issues, associate editor for ISIFs electronic Journal of Advances in Information Fusion, and is a member of the editorial board of IEEEs Signal Processing Magazine. He has been a member of the IEEE AESS Board of Governors since 2003. He was General Co- Chair (with Stefano Coraluppi) for the 2006 ISIF/IEEE Fusion Conference in Florence, Italy, Program Co-Chair (with Eugene Santos) for the 2003 IEEE Conference on Systems, Man, and Cybernetics in Washington DC, and Program Co-Chair (with Pramod Varshney) for the 1999 Fusion Conference in Sunnyvale. 